University of St.Gallen

\title{
Testing Conditional Asset Pricing Models Using a Markov Chain Monte Carlo Approach
}

Manuel Ammann

Michael Verhofen

Working Paper Series in Finance

Paper No. 21 


\title{
Testing Conditional Asset Pricing Models Using a Markov Chain Monte Carlo Approach
}

\author{
Manuel Ammann and Michael Verhofen* \\ University of St. Gallen
}

\begin{abstract}
We use Markov Chain Monte Carlo (MCMC) methods for the parameter estimation and the testing of conditional asset pricing models. In contrast to traditional approaches, it is truly conditional because the assumption that time variation in betas is driven by a set of conditioning variables is not necessary. Moreover, the approach has exact finite sample properties and accounts for errors-in-variables. Using S\&P 500 panel data, we analyze the empirical performance of the CAPM and the Fama and French (1993) three-factor model. We find that time-variation of betas in the CAPM and the time variation of the coefficients for the size factor (SMB) and the distress factor (HML) in the three-factor model improve the empirical performance. Therefore, our findings are consistent with time variation of firm-specific exposure to market risk, systematic credit risk and systematic size effects. However, a Bayesian model comparison trading off goodness of fit and model complexity indicates that the conditional CAPM performs best, followed by the conditional three-factor model, the unconditional CAPM, and the unconditional three-factor model.
\end{abstract}

\section{JEL: G12}

Keywords: Markov Chain Monte Carlo, Conditional Asset Pricing, Bayesian Analysis

\footnotetext{
*Manuel Ammann (manuel.ammann@unisg.ch) is professor of finance at the Swiss Institute of Banking and Finance, University of St. Gallen, Switzerland, and Michael Verhofen (verhofen@gmail.com) is visiting scholar at the Haas School of Business, University of California at Berkeley, United States of America. We thank an anonymous referee, the editor, Bernd Brommundt, Michael Genser, Alexander Ising, Stephan Kessler, Axel Kind, Angelika Noll, Ralf Seiz, Paul Söderlind, Stephan Süss, Evert Wipplinger, Rico von Wyss, and Andreas Zingg for valuable comments. We acknowledge helpful comments from the participants of the conference of the Swiss Society for Financial Market Research in Zürich in April 2005 and of the conference of the German Finance Association in Augsburg in October 2005. We acknowledge financial support from the Swiss National Science Foundation (SNF).
} 


\section{Introduction}

We use numerical Bayesian methods, i.e., Markov Chain Monte Carlo (MCMC) methods, for the estimation and the testing of unconditional and conditional asset pricing models. We estimate time-varying parameters for the CAPM and the Fama \& French (1993) three-factor model.

The empirical performance of the CAPM has been analyzed by a large number of authors, e.g., Fama \& French (1993), Jagannathan \& Wang (1996), Ferson \& Harvey (1999), and Heston, Rouwenhorst \& Wessels (1999). Various anomalies have been identified challenging the CAPM. Well-known anomalies include the size effect and ratios of the stock price to firm specific accounting numbers as earnings or the book value of equity, as shown by Basu (1977), Banz (1981), Chan, Hamao \& Lakonishok (1991), Fama \& French (1992) and Petrella (2005), among others. Fama \& French (1993) propose the so-called three-factor model, which uses, in addition to market risk, two additional risk factors to explain asset pricing anomalies. The three-factor model uses the difference in returns between small and big stocks, referred to as the small-minus-big (SMB) risk factor, and the difference in returns between stock with a high book-to-market ratio and stocks with a low book-to-market ratio, referred to as the high-minus-low (HML) factor, as additional explanatory variables. In general, the SMB factor is interpreted as a size premium and the HML factor as a value premium.

However, several investigations, such as Ferson \& Harvey (1991), Ferson \& Koraczyk (1995), Braun, Nelson \& Sunier (1995) and Koutmos \& Knif (2002), indicate that betas exhibit considerable time variation. Hansen \& Richard (1987) show that conditional versions of asset pricing models and, in particular, the CAPM can hold even when unconditional asset pricing models exhibits serious pricing errors.

Jagannathan \& Wang (1996) examine the empirical performance of the conditional CAPM in a cross section of stock returns. Overall, they find that the dynamic CAPM can explain the cross section of stock returns much better than a static CAPM. However, their approach has a number of shortcomings. Probably the most important shortcoming is the use of proxy variables for conditional betas and for other variables. In particular, they decompose the beta in a constant part, in a part related to the market return, and a 
part related to the credit spread between high-grade and low-grade bonds. The choice of conditioning variables is not investigated in detail. However, their approach is unable to detect non-linear behavior, firm-specific effects, and effects that cannot be explained by other variables. To account for errors-in-variables, they show how to adjust the confidence interval.

Bayesian tests of asset pricing models have a long history in finance. Shanken (1987) proposes a Bayesian approach to testing portfolio efficiency and derives a computationally convenient posterior-odds ratio. He shows that classical tests such as the approach by Gibbons, Ross \& Shanken (1989) might deliver wrong signals from a Bayesian point of view. He recommends using significance levels higher than 95\%. The Bayesian asset pricing test proposed by Harvey \& Zhou (1990) uses a Monte Carlo version of the approach by Shanken (1987). They use Monte Carlo integration to compute posterior-odds ratios for industry portfolios. They find that the probability that the portfolio is efficient is small. In other words, they can reject the CAPM. McCulloch \& Rossi (1991) extend previous approaches to multi-factor models. They compute posterior-odds ratios via the Savage density for the restricted and unrestricted versions and finally reject the APT model under consideration. Similarly, Geweke \& Zhou (1996) use Gibbs sampling to test restrictions. Avramov \& Chao (2006) have proposed an exact finite sample Bayesian test with conditioning information, i.e., they assume that time variation in factor loadings is driven by a set of conditioning variables.

The limitations of previous non-Bayesian approaches of conditional asset pricing models can be summarized as follows. First and probably most importantly, the use of conditioning variables for modelling variation of betas is a drawback, as Ghysels (1998) notes, because different specifications might lead to completely different results. For example, the use of a set of macroeconomic variables and the use of firm-specific variables as conditioning variables delivers different results. Second, two-step procedures ignore the fact that betas are estimates and, therefore, do not account for errors-in-variables, as Berglund \& Knif (1999) show. Third, most asset pricing tests, such as GMM approaches, rely on asymptotic estimation, possibly leading to biases in finite samples, as Ferson \& Foerster (1994) point out. Compared to the most recent conditional Bayesian test by Avramov $\&$ Chao (2006), we are also able to drop the assumption that time-variation in factor 
loadings is driven by a set of conditioning variables.

In this paper, we use Markov Chain Monte Carlo (MCMC) methods for estimating and testing conditional versions asset pricing models. This approach has a number of advantages over approaches previously used. A first, but very important feature is that we do not impose any restrictive assumptions about the behavior of betas, i.e., we do not assume that stock betas depend on specific variables, but treat the beta as a latent variable. Being more general, this approach is able to capture traditional approaches that use conditioning variables, but not vice versa. The estimation procedure can be regarded as a filter for extracting the unknown signal, i.e., the beta coefficients, from noisy data, i.e., the return series. And indeed, MCMC approaches can be regarded as a generalization of the Kalman filter, as pointed out by Robert \& Casella (1999). Second, our approach accounts for estimation risk, i.e., the so-called errors-in-variables problem does not exist. Third, the MCMC estimation procedure has exact finite sample properties because it does not rely on asymptotic estimators, as noted by Zellner (1971). Fourth, the MCMC approach can also account for model uncertainty.

Using the MCMC approach, we estimate the unconditional and conditional CAPM and the Fama \& French (1993) three-factor model. The econometric analysis of S\&P 500 panel data delivers new insights compared to existing approaches, in particular in relation to the approaches by Jagannathan \& Wang (1996), Ferson \& Harvey (1999) and Wang (2003). First, the empirical performance of the conditional CAPM is slightly better than the empirical performance of the unconditional CAPM, measured by $R^{2}$. Second, for the conditional and unconditional Fama \& French (1993) three-factor model, the variance explained increases from $28.0 \%$ to $31.7 \%$. In particular, our analysis shows that the factor loadings for the high-minus-low (HML) and small-minus-big (SMB) factor vary over time. These findings indicate that the exposure of individual stocks to market risk, systematic size effects and credit risk depends heavily on the observed time period. Third, a Bayesian model comparison using the so-called Bayes factors indicates that the conditional CAPM performs better than the unconditional CAPM. Additionally, there is evidence that the unconditional three-factor model is misspecified compared to both versions of the CAPM. From a Bayesian perspective, the conditional three-factor model has a posterior chance of 1.61 to 1 of being true compared to the unconditional CAPM and 
of 2.41 to 1 compared to the unconditional three-factor model. However, the posterior odds of the conditional three-factor model to the conditional CAPM is 0.44 to 1 . Therefore, from a Bayesian model selection perspective, the conditional three-factor model is inferior to the conditional CAPM.

The article is structured as follows. In Section 2, we outline the Bayesian approach for the estimation of conditional asset pricing models. In Section 3, we present our empirical results. Section 4 concludes.

\section{Estimation of Parameters for Conditional Asset Pricing Models}

In this section, the setup of the dynamic-factor asset pricing model and the Bayesian estimation method is presented.

\subsection{A Dynamic Factor Asset Pricing Model}

The general form of a conditional linear asset pricing model is given by

$$
\mathbb{E}_{t-1}\left[r_{n, t}^{e}\right]=\mathbb{E}_{t-1}\left[\sum_{k=1}^{K} \beta_{k, n, t} \Psi_{k, t}\right]
$$

where $r_{n, t}^{e}$ is the excess return for an asset $n$ at time $t$ over the risk free rate, $\beta_{k, n, t}$ the exposure to a risk factor paying the risk premium $\Psi_{k, t}$, and $K$ denotes the number of risk factors. $\mathbb{E}_{t-1}$ denotes the expectation conditional on the information set at time $t-1$. Assuming that all risk factors and all risk premia are uncorrelated with each other and that the estimate for beta at time $t-1$ is the best unbiased forecast for beta at time $t$, we obtain

$$
\begin{aligned}
\mathbb{E}_{t-1}\left[r_{n, t}^{e}\right] & =\sum_{k=1}^{K} \mathbb{E}_{t-1}\left[\beta_{k, n, t}\right] \mathbb{E}_{t-1}\left[\Psi_{k, t}\right] \\
& =\sum_{k=1}^{K} \beta_{k, n, t-1} \mathbb{E}_{t-1}\left[\Psi_{k, t}\right]
\end{aligned}
$$

Because of unobservability of $\mathbb{E}_{t-1}\left[\Psi_{k, t}\right]$, we replace $\mathbb{E}_{t-1}\left[\Psi_{k, t}\right]$ with the realized risk 
premium $\Psi_{k, t}$ for testing purposes. Therefore, the testing of a conditional asset pricing model requires the equation

$$
r_{n, t}^{e}=\sum_{k=1}^{K} \beta_{k, n, t-1} \Psi_{k, t}+u_{n, t}
$$

to be estimated. ${ }^{1}$ The error terms $u_{n, t}$ are assumed to have zero mean and variance $\sigma_{u, n, t}^{2}$.

In contrast to previous approaches, we do not assume that time varying betas depend on a set of conditioning variables. We allow for time variation in the coefficients by assuming that the coefficients follow a random walk:

$$
\beta_{k, n, t}=\beta_{k, n, t-1}+v_{k, n, t}
$$

where the error terms $v_{k, n, t}$ have zero mean and variance $\sigma_{v, k, n, t}^{2}$. This random walk model is very general because it is able to cover a wide range of gradual coefficient variation reasonably well. ${ }^{2}$

However, to ensure identifiability of the model, it is necessary to impose restrictions on the variance of the error terms $u$ and $v$. The motivation for such a restriction is rather intuitive: the variance of a signal, i.e., the betas $\beta_{n, k, t}$, must be less than the variance of the observation equation, i.e., the time series of returns $r_{n, t}^{e}$. In a general state space context, this was first documented by Akaike (1980).

The noise-to-signal ratio, $\lambda_{k, n, t}^{2}$, representing the relationship between $\sigma_{v, k, n, t}^{2}$ and $\sigma_{u, n, t}^{2}$, is defined as

$$
\lambda_{k, n, t}^{2}=\frac{\sigma_{u, n, t}^{2}}{\sigma_{v, k, n, t}^{2}} .
$$

The noise-to-signal ratio, $\lambda^{2}$, must be at least one. Higher values of $\lambda^{2}$ correspond to greater smoothing. The parameter $\lambda$ has a rather straightforward interpretation, pointed out by Kitagawa \& Gersch (1996): the smoothing parameter $\lambda$ determines the relative weight for the goodness of fit versus smoothness. 


\subsection{Bayesian Inference}

For the estimation of the linear factor model with time-varying coefficients, a Bayesian estimation approach is used. In classical approaches such as maximum likelihood, inference is based on the likelihood of the data alone. In contrast, in Bayesian inference, the likelihood of the observed data $D$ given parameters $\theta$, denoted as $\mathrm{L}(D \mid \theta)$, is used to modify the prior beliefs about the parameter set $\mathrm{P}(\theta)$. The updated knowledge is summarized in a posterior density $\mathrm{P}(\theta \mid D)$, as noted by Robert \& Casella (1999). Formally, Bayes theorem is used to determine the posterior distribution of $\theta$ conditional on $D$ :

$$
\mathrm{P}(\theta \mid D)=\frac{\mathrm{L}(D \mid \theta) \mathrm{P}(\theta)}{\mathrm{P}(D)},
$$

where the marginal distribution of the data $\mathrm{P}(D)$ is given by

$$
\mathrm{P}(D)=\int \mathrm{L}(D \mid \theta) \mathrm{P}(\theta) d \theta \quad .
$$

The posterior distribution of the parameters $\mathrm{P}(\theta \mid D)$ is the object of Bayesian inference. Bayesian inference therefore requires three steps. First, the prior distributions are defined. Second, the likelihood function depending on the structural equations for the model is determined. Third, the posterior distribution is computed.

Bayesian inference in econometrics has at least four main advantages. First and probably most importantly, Bayesian econometric methods can be used to estimate parameters for almost any model. Second, Bayesian inference nests all classical estimation methods such as maximum likelihood if priors are uninformative. Third, if priors are informative, Bayesian inference provides a formal procedure for incorporating prior information. Fourth, Bayesian inference does not rely on asymptotic properties of the estimators, but has exact finite-sample properties, as noted by Zellner (1971).

The Metropolis-Hastings algorithm proposed by Metropolis \& Ulam (1949) and extended by Hastings (1970) has basically two functions. First, the algorithm maximizes the posterior distribution $\mathrm{P}(\theta \mid D)$. Second, after convergence, the Metropolis-Hastings algorithm creates a sample of parameters vectors needed to draw conclusions about the parameters.

The main idea behind this algorithm is that at each iteration $i$ the next state $\theta_{i+1}$ is 
chosen by sampling a candidate point $\theta_{c}$ from a proposal density $q\left(. \mid \theta_{i}\right)$. The candidate point $\theta_{c}$ is accepted with probability $\alpha\left(\theta_{i}, \theta_{c}\right)$, where

$$
\alpha\left(\theta_{i}, \theta_{c}\right)=\min \left(1, \frac{\mathrm{P}\left(\theta_{c} \mid D\right) q\left(\theta_{i} \mid \theta_{c}\right)}{\mathrm{P}\left(\theta_{i} \mid D\right) q\left(\theta_{c} \mid \theta_{i}\right)}\right)
$$

If the candidate point is accepted, the next state becomes $\theta_{i+1}=\theta_{c}$, otherwise $\theta_{i+1}=$ $\theta_{i}$. The Metropolis-Hastings-algorithm can be illustrated by the following pseudo code:

\author{
Choose $\theta_{0}$, Set $i$ to 0 , \\ Select number of iterations $a$ \\ Do while $i<a$ \\ Sample a point $\theta_{c}$ from $q\left(. \mid \theta_{i}\right)$ \\ Sample a Uniform $(0,1)$ random variable $U$ \\ If $U$ is smaller than $\alpha\left(\theta_{i}, \theta_{c}\right)$, set $\theta_{i+1}$ to $\theta_{c}$ \\ otherwise set $\theta_{i+1}$ to $\theta_{i}$ \\ Increase i
}

Repeat

This algorithm accepts a new parameter set if the new parameter set increases the posterior density. If the new parameter set decreases the posterior density, the new parameter set is accepted with a specific probability.

For the proposal density, a number of choices are possible (see e.g. Robert \& Casella $(2005))$. We have set $\alpha\left(\theta_{i}, \theta_{c}\right)=1$, i.e., new draws are always accepted. In this case, the Metropolis-Hastings algorithm is a Gibbs sampler (Geman \& Geman (1984)) or a version of Gibbs sampling such as slice updating (Neal (2003)). Kim \& Nelson (1999) and Congdon (2003) show how to implement dynamic factor models using Gibbs sampling.

The Metropolis-Hastings algorithm creates a sample of parameter draws that can be used to derive conclusions about the parameters. The posterior expectation of a function of the parameters $f(\theta)$, such as the mean and the standard deviation (standard error) of the parameters, is given by 


$$
\mathbb{E}[f(\theta) \mid D]=\frac{\int f(\theta) \mathrm{P}(\theta) \mathrm{P}(D \mid \theta) d \theta}{\int \mathrm{P}(\theta) \mathrm{P}(D \mid \theta) d \theta}
$$

To evaluate the posterior expectation of a function of the parameters $f(\theta)$, Monte Carlo integration is applied. Monte Carlo integration evaluates $\mathbb{E}[f(\theta)]$ by drawing samples $\left\{\theta_{t}, t=1, \ldots, n\right\}$ from $\mathrm{P}(\theta \mid D)$ and then approximating the expectation

$$
\mathbb{E}[f(\theta)] \approx \frac{1}{a} \sum_{i=1}^{a} f\left(\theta_{i}\right)
$$

This approximation is feasible if a Markov Chain with $\mathrm{P}(\theta \mid D)$ as its stationary distribution is used. A Markov Chain is a sequence of random variables $\left\{\theta_{0}, \theta_{1}, \theta_{2}, \ldots\right\}$ such that the next state $\theta_{i+1}$ sampled from the distribution (or transition kernel) $\mathrm{P}\left(\theta_{i+1} \mid \theta_{i}\right)$ only depends on the current state and not on the entire history. Robert \& Casella (1999) show that the Metropolis-Hastings algorithm creates such a stationary distribution. This procedure using Monte Carlo integration and Markov Chains is called Markov Chain Monte Carlo (MCMC).

As the starting value $\theta_{0}$ affects the distribution in Monte Carlo simulations, a sufficiently large number of burn-in iterations is discarded from the sample, assuming that $\mathrm{P}^{(i)}\left(. \mid \theta_{0}\right)$ does not depend on $i$ or $\theta_{0}$. An estimator for the expectation $\mathbb{E}[f(\theta)]$ is given by a sample average of the parameter sample generated by the Metropolis-Hastings algorithm, i.e.,

$$
\bar{f}(\theta)=\frac{1}{a-b} \sum_{i=b+1}^{a} f\left(\theta_{i}\right),
$$

where $a$ is the total number of iterations and $b$ the length of the burn-in period.

\subsection{Priors, Likelihood and Posterior}

Based on the asset pricing model derived previously and assuming that errors are normally distributed, we define the following structural equations 


$$
\begin{gathered}
r_{n, t}^{e} \sim \mathcal{N}\left(\sum_{k=1}^{K} \beta_{k, n, t} \Psi_{k, t}, \sigma_{u, n}\right) \quad \text { for } n=\{1, \ldots, N\}, t=\{2, \ldots, T\} \\
\beta_{k, n, t} \sim \mathcal{N}\left(\beta_{k, n, t-1}, \sigma_{v, k, n}\right) \text { for } k=\{1, \ldots, K\}, n=\{1, \ldots, N\}, t=\{2, \ldots, T\},
\end{gathered}
$$

where $\mathcal{N}$ denotes a normal distribution. The extension to a non-normal setting remains for further research (e.g. Carlin, Polson \& Stoffer (1992)). As prior distributions, we use uninformative priors

$$
\begin{aligned}
\sigma_{u, n}^{2} & \sim \operatorname{Uniform}\left(0, M_{\sigma}\right) \text { for } n=\{1, \ldots, N\} \\
1 / \lambda_{k}^{2} & \sim \operatorname{Uniform}(0,1) \text { for } k=\{1, \ldots, K\} \\
\beta_{k, n, 1} & \sim \mathcal{N}\left(0, M_{\beta}\right) \quad \text { for } k=\{1, \ldots, K\} \text { and } n=\{1, \ldots, N\}
\end{aligned}
$$

where $\sigma_{v, m, n}^{2}=\sigma_{u, n}^{2} / \lambda_{m}^{2}, N$ denotes the number of stocks, $K$ the number of risk factors and $T$ the length of the time series. Uniform denotes a uniform distribution. For $M_{\sigma}$ and $M_{\beta}$, high values have been chosen $\left(M_{\sigma}=600 \text { (per month), } M_{\beta}=10\right)^{3}$.

We assume constant volatility. Bauwens, Lubrano \& Richard (1999) note that heteroscedasticity introduces an inconsistency of the estimator of the variance of the ordinary least square estimator, i.e., estimated standard errors are too small, but the estimator itself is unbiased. The assumption of constant volatility follows many other approaches for testing capital asset pricing models, such as Fama \& MacBeth (1973), and Gibbons et al. (1989).

The likelihood function can be computed by multiplying the distributions for the excess returns $r_{n, t}^{e}$ conditional on the factor loadings $\beta_{k, n, t}$ and the distribution for the factor loadings $\beta_{k, n, t}$ conditional on the factor loadings in the previous period $\beta_{k, n, t-1}$. Therefore, the complete likelihood function is given by 


$$
\begin{gathered}
\mathrm{L}=\prod_{n=1}^{N}\left(\frac{1}{2 \pi \sigma_{u, n}^{2}}\right)^{T / 2} \exp \left[-\frac{1}{2 \sigma_{u, n}^{2}} \sum_{t=2}^{T}\left(r_{n, t}^{e}-\sum_{k=1}^{K} \beta_{k, n, t-1} \Psi_{k, t}\right)^{2}\right] \\
\prod_{k=1}^{K} \prod_{n=1}^{N}\left(\frac{1}{2 \pi \sigma_{v, k, n}^{2}}\right)^{T / 2} \exp \left[-\frac{1}{2 \sigma_{v, k, n}^{2}} \sum_{t=2}^{T}\left(\beta_{k, n, t}-\beta_{k, n, t-1}\right)^{2}\right] .
\end{gathered}
$$

In the empirical part, we report the posterior mean (the sample average of the Markov Chain) for each parameter and its standard error (the sample standard deviation of the Markov Chain) for each parameter.

The posterior distribution $\mathrm{P}(\theta \mid D)$, which is the product of the likelihood function and all prior distributions, is therefore proportional to

$$
\mathrm{P}(\theta \mid D) \propto \mathrm{L} \cdot \prod_{n=1}^{N} \frac{1}{M_{\sigma}} \prod_{k=1}^{K}\left(\frac{1}{2 \pi M_{\beta}^{2}}\right)^{N / 2} \exp \left[-\frac{\beta_{k, n, 1}^{2}}{2 M_{\beta}^{2}}\right]
$$

\subsection{Bayes Factors}

Jeffreys (1961) introduced and defined the so-called Bayes factors for the testing of hypotheses in a Bayesian context. The Bayes factor B, which tests two models $M_{1}$ and $M_{0}$ against each other, is defined as the posterior to prior odds ratio of the relevant models, i.e.,

$$
\mathrm{B}_{1,0}=\frac{\mathrm{P}\left(M_{1} \mid D\right)}{\mathrm{P}\left(M_{0} \mid D\right)}=\frac{\mathrm{P}\left(D \mid M_{1}\right)}{\mathrm{P}\left(D \mid M_{0}\right)} \frac{\mathrm{P}\left(M_{0}\right)}{\mathrm{P}\left(M_{1}\right)}
$$

where $\mathrm{P}(D \mid M)$ denotes the marginal likelihood of the data $D$ given a model $M$ and $\mathrm{P}(M)$ the prior probability for a model $M$.

As noticed by Denison, Holmes, Mallick \& Smith (2002), the marginal likelihood of a model $M$ gives a measure of the probability of observing the data given that $M$ is true. If the marginal likelihood is high, it is more likely that the observed time series was generated by the assumed model. However, similar to conventional likelihood ratio tests, the Bayes factor expresses the relative support given by the data for each model, as Kass \& Raftery (1995) note.

Bayes factors are very powerful because they balance the goodness of fit against the 
complexity of a model (see e.g. Denison et al. (2002) and MacKay (2003)) and, therefore, obey the Occam's razor. The Occam's razor is a principle that states a preference for simple theories and states that the simplest explanation that fits the data should be accepted.

Figure 1 illustrates the logic behind Bayes factors. Suppose there are two competing models, denoted as $M_{0}$ and $M_{1}$, where model $M_{0}$ is more complex and, therefore, is able to provide a better goodness of fit, ceteris paribus. Suppose next that we have observed the point 0 in the data space. Then, the ratio of the marginal likelihoods, i.e., the Bayes factors, for $M_{1}$ and $M_{0}$ at point 0 is 3.4. Therefore, for $D$ equal to 0 , we should favor the simpler model $M_{1}$. However, for $M$ equal to 1 , the probability that the simple model $M_{1}$ can explain the data is very small and therefore, the Bayes factor is very high and favors the complex model $M_{0}$. 


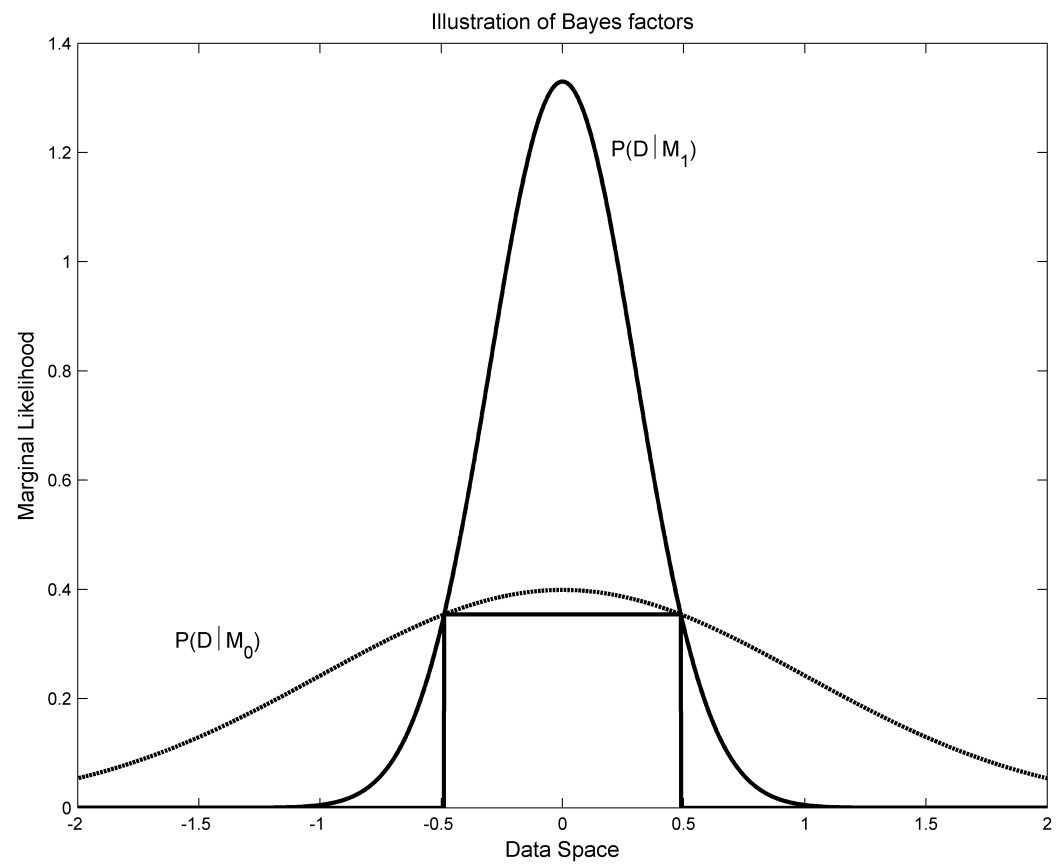

Figure 1: Illustration of Bayes Factors

The figure illustrates how Bayesian inference adheres to the principle of Occam's razor. Similar to Denison et al. (2002) and MacKay (2003), the horizontal axis represents the data-space with the vertical axis giving the marginal likelihood of the data. Suppose there are two competing models $M_{0}$ and $M_{1}$, where $M_{1}$ covers only a subspace of the space covered by $M_{0}$. If the observed data are in the region -0.5 to 0.5 , then the simpler model $M_{1}$ is preferred as it has a higher probability in this region. 
Bayes factors can be used for two purposes. First, they can be used to test an unrestricted model $M_{0}$ against its restricted version $M_{1}$. In such a setting, the Bayes factors are similar to likelihood ratio tests. Second, they can be used to test two competing models $M_{1}$ and $M_{0}$ against each other. Therefore, they enable to account for model uncertainty. In particular, this is important if the correct specification of a model is unknown. The so-called Bayesian model averaging relies on Bayes factors to compute posterior odds for different models.

Bayes factors have a rather intuitive interpretation. Suppose a researcher would assign uninformative, equal prior probabilities to $M_{1}$ and $M_{0}$, i.e., $50 \%$ for each model. After having seen the data, the Bayes factor shows how to adjust the prior probabilities to compute posterior probabilities for the models, e.g., a Bayes factor of 4 indicates that the researcher should assign a posterior probability for $M_{1}$ that is four times higher than for $M_{0}$, i.e. $80 \%$ for model $M_{1}$ and $20 \%$ for model $M_{0}$.

The interpretation proposed by Jeffreys (1961) is still the standard. Jeffreys (1961) shows that Bayes factors of less than 1 support the model $M_{0}$ and Bayes factors larger than 1 support model $M_{1}$. He interprets a Bayes factor between 1 and 3 as weak support for $M_{1}$, between 3 to 20 as support for $M_{1}$, between 20 and 150 as strong evidence for $M_{1}$ and over 150 as very strong support for $M_{1}$. However, the calculation of Bayes factors is a challenging task, as Han \& Carlin (2001) note, because the computation of Bayes factors requires, in general, the evaluation of the marginal likelihood. Analytical solutions are only available for a very limited number of cases.

In this study, the widely used approach by Carlin \& Chib (1995) is applied. Carlin \& Chib (1995) propose a two-step procedure for the computation of the Bayes factor. In a first step, they estimate the parameters for each model separately using MCMC methods. In a second step, they iterate over the model space. In particular, they take the parameters including their confidence intervals as given and, using MCMC methods, they try to find the optimal mixture ratio for a set of models.

\subsection{Monte Carlo Study}

Since Bayesian inference is less well explored than more traditional econometric approaches, we check for the reliability of the estimators. First, we construct artificial 
data sets based on the data generating process specified in equations 4 to 6 . Second, using these artificial data sets, we estimate the parameter using the Metropolis-Hastings algorithm and Markov Chain Monte Carlo (MCMC) methods. Third, we compare the real parameters of our artificial data set and estimated parameters.

In our approach, the data generating process is given by the equations 4,5 , and 6. Therefore, we have to specify the volatility of the return for each single stock, the smoothing parameter for each risk factor determining the volatility of the risk factor in relation to the volatility of the return for each single stock, and the starting values for each risk factor and for each stock. As a general rule for the construction of the artificial data sets, we create artificial data sets similar to the original data set used in our empirical analysis. For evaluation purposes, we use the most general asset pricing model, i.e., the conditional three-factor model. As starting values, we choose a wide range of different parameters and parameter combinations to capture all realistic combinations. In particular, as starting values for the beta coefficients, we use values between 0.6 and 1.4. For the factor loadings on the HML and SMB factor, we choose different starting values around 0 covering most of the estimated parameters in the original data set. In particular, we choose values between -0.4 and 0.4 for factor loadings on the HML and SMB factor. The volatility is set to values between 5 and 10 per month. The smoothing parameter is set to $1.0 \%$ for the volatility of the beta factor, to $1.5 \%$ for the volatility for the HML factor and to $0.5 \%$ for the SMB factor.

In total, we create 100 artificial data sets by using the predefined starting values and drawing random numbers for simulating the process for the factor loadings and the return process. Using this artificial return data, we estimate the parameters for the factor loadings for each risk factor and for each stock.

A comparison of the true factor loadings and estimated factor loadings show that the estimation procedure is able to recover the data generating process correctly and precisely.

Moreover, for the unconditional models, we countercheck the estimated coefficients with standard OLS beta estimates. The difference between the beta values estimated by the MCMC method and by OLS is negligible and only occurs after the second decimal point. This result is not surprising since the assumptions in the constant CAPM and in the constant three-factor model are equal to standard OLS assumptions. However, it is 
an indication for the functionality of the method and its implementation.

\section{Empirical Results}

\subsection{Data and Implementation}

For the analysis, we use the S\&P 500 index constituents per August 2004 in the time period from January 1974 to August 2004. ${ }^{4}$ The data set is from Datastream. Monthly data have been used for the analysis. Our sample consists of 500 stocks and contains 368 months of return data. The data for the market portfolio (MRP), the high-minus-low (HML) factor and the small-minus-big (SMB) factor is from the Fama and French data library. For the calculation of these factors we refer to Fama \& French (1993). As the risk-free rate we use the 3 -month US Treasury bill rate on the secondary market. The data is from the Federal Reserve Bank.

In contrast to previous studies, we use individual stocks instead of portfolios of stocks for our analysis. Since the purpose of this study is to capture firm-specific, time-varying effects, we focus on individual stocks. Moreover, factor loadings for individual stocks are, from an economic point of view, more easily interpretable. In total, we test four different models: (1) unconditional CAPM, (2) conditional CAPM, (3) unconditional three-factor model and (4) conditional three-factor model.

The implementation has been carried out using the software package "Bayesian Analysis Using Gibbs Sampling" (WinBUGS). Given the priors and the likelihood, coding is straightforward. This way of implementation has been chosen to make the results reproducible and because the purpose of this study is not the development of new sampling algorithms. Standard samplers as presented in Robert \& Casella (2005) are immediately applicable. In particular, a slice sampler (Neal (2003)) has been used, although other samplers are probably also applicable. For a discussion of Bayesian statistical packages in general we refer to Carlin \& Louis (2000) and for WinBUGS in particular, we refer to Congdon (2001), Congdon (2003) and Cowles (2004). 

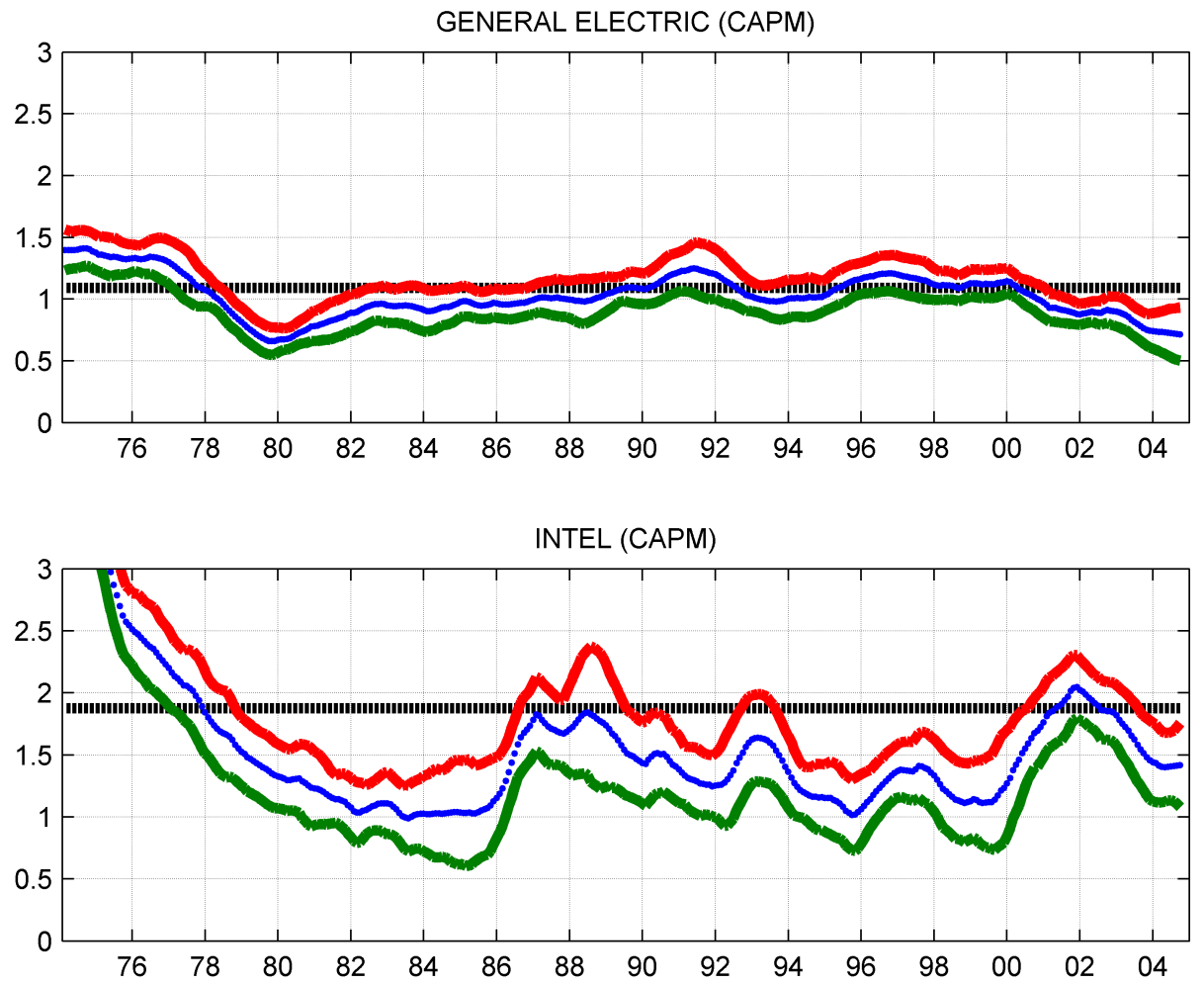

Figure 2: Factor loading for Intel and General Electric in the unconditional and the conditional CAPM

The figure shows the factor loading for Intel and General Electric in the unconditional and the conditional CAPM. The horizontal line shows the unconditional beta. The remaining three lines show the conditional beta (middle line) and the one-sigma confidence interval (between the upper and the lower line).

\subsection{Conditional vs. Unconditional CAPM}

Figure 2 shows the estimated beta coefficients for the unconditional CAPM and the conditional CAPM for General Electric (GE) and Intel over the sample period from 1974 to 2004. The constant beta is indicated by a horizontal line. The two stocks have been selected to illustrate the very different behavior of betas. The two stocks can also be regarded as typical representatives of two different types of companies, namely a large diversified company and a very focused technology stock. 
The beta for General Electric shows a rather stable behavior in the sample period in comparison to other stocks. The conditional beta fluctuated around the unconditional beta. In contrast, Intel's beta shows a much higher time variation and much wider confidence intervals. Between 1974 and 1982, Intel's beta shows a strong downward trend. For the period 1986 to 2004, there is evidence for some cyclical movements in the beta. The recessions in the sample period ${ }^{5}$ have no visible impact on the factor loading.

Similar figures for selected companies can be found in the appendix. Figures A1 to A6 show the estimated beta values with constant and time-varying coefficients over the sample period from 1974 to 2004 for selected stocks from the cross section. In general, there is no unique pattern in the time variation of estimated betas, but some patterns such as trending or stable behavior repeat themselves.

Large and established companies such as Exxon, General Motors and American Electric Power show a remarkably stable behavior around the constant beta estimation. Strong cyclical behavior around the constant beta estimate such as for Aflac seems to be the exception. A number of smaller and less well established companies such as Family Dollar Stores show a strong downward trending behavior.

\subsection{Conditional vs. Unconditional Three-Factor Model}

Figure 3 shows the estimated factor loadings for the market risk, the HML factor, and the SMB factor for General Electric and Intel. The comparison of the conditional betas in the conditional CAPM and in the conditional three-factor model shows that the results are very similar. This observation can also be made for the whole cross section. 


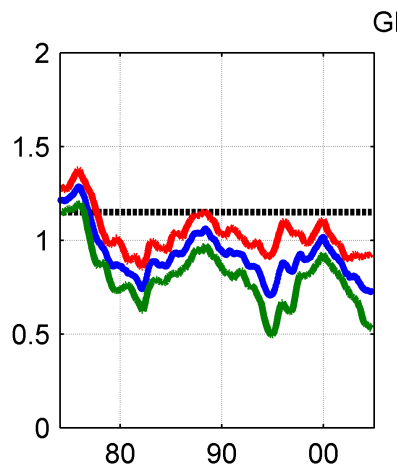

GENERAL ELECTRIC (MRP, HML, SMB)
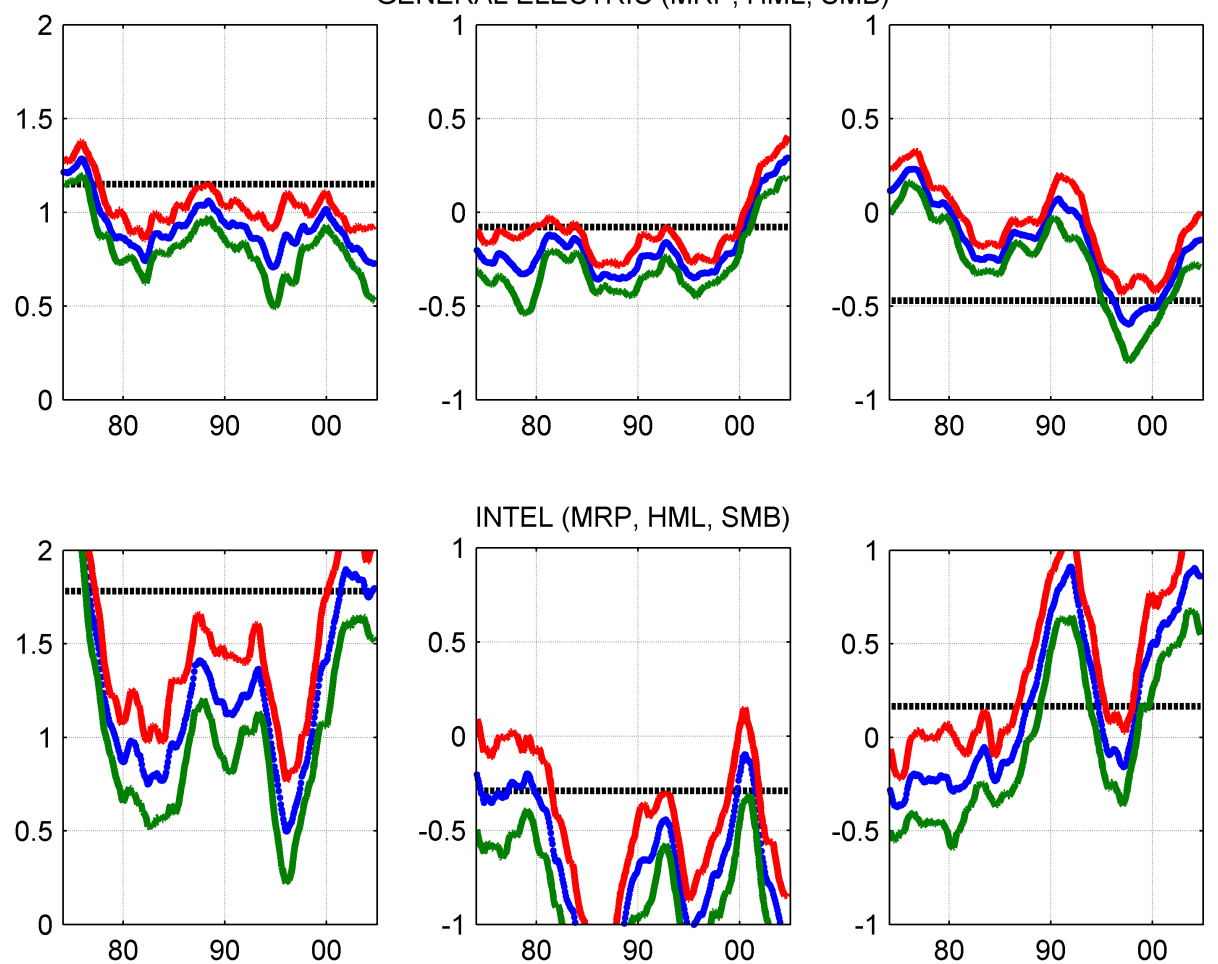

Figure 3: Factor loadings for Intel and General Electric in the unconditional and the conditional Fama and French three-factor model

The figure shows the factor loadings on the market risk premium (MRP), the value premium (HML), and the size factor (SMB) for Intel and General Electric in the unconditional and the conditional (including estimated standard error) Fama and French three-factor model. 
In general, the coefficients for the HML and SMB factor show, for all firms in the sample including the examples General Electric and Intel, a variance and time variation similar to the betas. For General Electric, the unconditional HML factor is slightly negative. This is an indication that General Electric is more of a growth stock than a value stock. The conditional HML factor does not exhibit a clear time trend until 2000 and is statistically not different from the unconditional HML factor at a 95\% confidence level. However, the SMB factor shows a different behavior. The unconditional SMB factor is negative, indicating that General Electric is a large firm. This is, of course, consistent with prior expectations. The conditional SMB coefficient shows a downward trend from +0.2 in 1974 to -0.6 in 1998, which is consistent with GE's increase in market capitalization.

For Intel, the HML and SMB factors show a very different behavior. Whereas the unconditional HML is around -0.3 (meaning that Intel is a growth stock), the conditional HML shows large variations. For Intel, both the unconditional and conditional SMB factor show a positive exposure to the systematic small size effect. This is an indication that the stock returns for Intel behave more like those of a small firm than those of a large firm. Again, time variation is substantial.

Similar figures for an extended sample of stocks can be found in the appendix. Figures A1 to A6 show the estimated factor loadings in the conditional and unconditional threefactor model. The comparison of the constant and the time-varying HML and SMB factor shows that both factors exhibit a considerable variation during time. Deviations of the time-varying HML and SMB factor from the constant HML and SMB factor are, in general, persistent and substantial. However, this result is not surprising if we regard the HML factor as a proxy variable for value and growth stocks and the SMB factor as a proxy variable for the size effect. In general, for value stocks, the HML factor should be positive and for growth stocks negative. Large and mature companies should show a negative SMB factor since their exposure to the systematic small company effect should be negative. Most findings are consistent with this prior expectation.

Repeating patterns across different companies can hardly be found. Individual, firmspecific effects seem to play an important role. However, this result is not surprising since the characterization of a firm as a value stock (HML) and the firm size (SMB) is, similar to the exposure to market risk, a firm specific risk factor. Over the whole sample period a 
number of firms such as AT\&T, American Electric Power, Dow Jones, Exxon Mobil and Procter\&Gamble show a very small and roughly constant exposure to systematic value effects and size effects.

Concerning the HML factor, most stocks show a positive loading on the HML factor, i.e., they are classified as value stocks. Overall, we find that companies facing financial distress have a strong positive loading on the HML factor. For a number of companies the factor loading has changed substantially since 2000 perhaps indicating a decrease in credit worthiness (such as American Airlines, Baxter and Cummins).

Overall, our findings are consistent with Fama and French (1998), who regard the HML factor as a proxy for value firms and firms under financial distress for positive factor loadings and as a proxy for growth companies for negative factor loadings.

For the SMB factor, we expect negative coefficients for large firms and positive coefficients for small firms. However, the evidence is not as clear as for the HML factor. One typical example is the estimated SMB loading for WalMart. During the sample period, WalMart and its market capitalization has grown substantially. This development is also reflected is the estimated SMB coefficient, which is positive at the start of the sample period and negative at the end of the sample period.

\subsection{Model Comparison}

For model comparison, we use three different measures. We start with traditional measures of the explanatory power of a model, such as $R^{2}$ and root mean squared error (RMSE). Then, we turn to Bayesian model comparison criteria.

Figure 4 shows the estimated $R^{2}$ for the whole sample period and over a rolling horizon of 60 months. Over the whole sample period, the $R^{2}$ of the CAPM with time-varying betas is $27.97 \%$. It is higher than the $R^{2}$ of the unconditional CAPM, which has an $R^{2}$ of $23.63 \%$. The explanatory power of the unconditional three-factor model $\left(R^{2}\right.$ of $27.95 \%$ ) is almost identical to the conditional CAPM, whereas the explanatory power of the conditional three-factor model increases to $31.69 \%$. Our conclusion is that taking firm-specific, time-varying market risk, credit risk, and size effects into account improves the performance of asset pricing models slightly. 

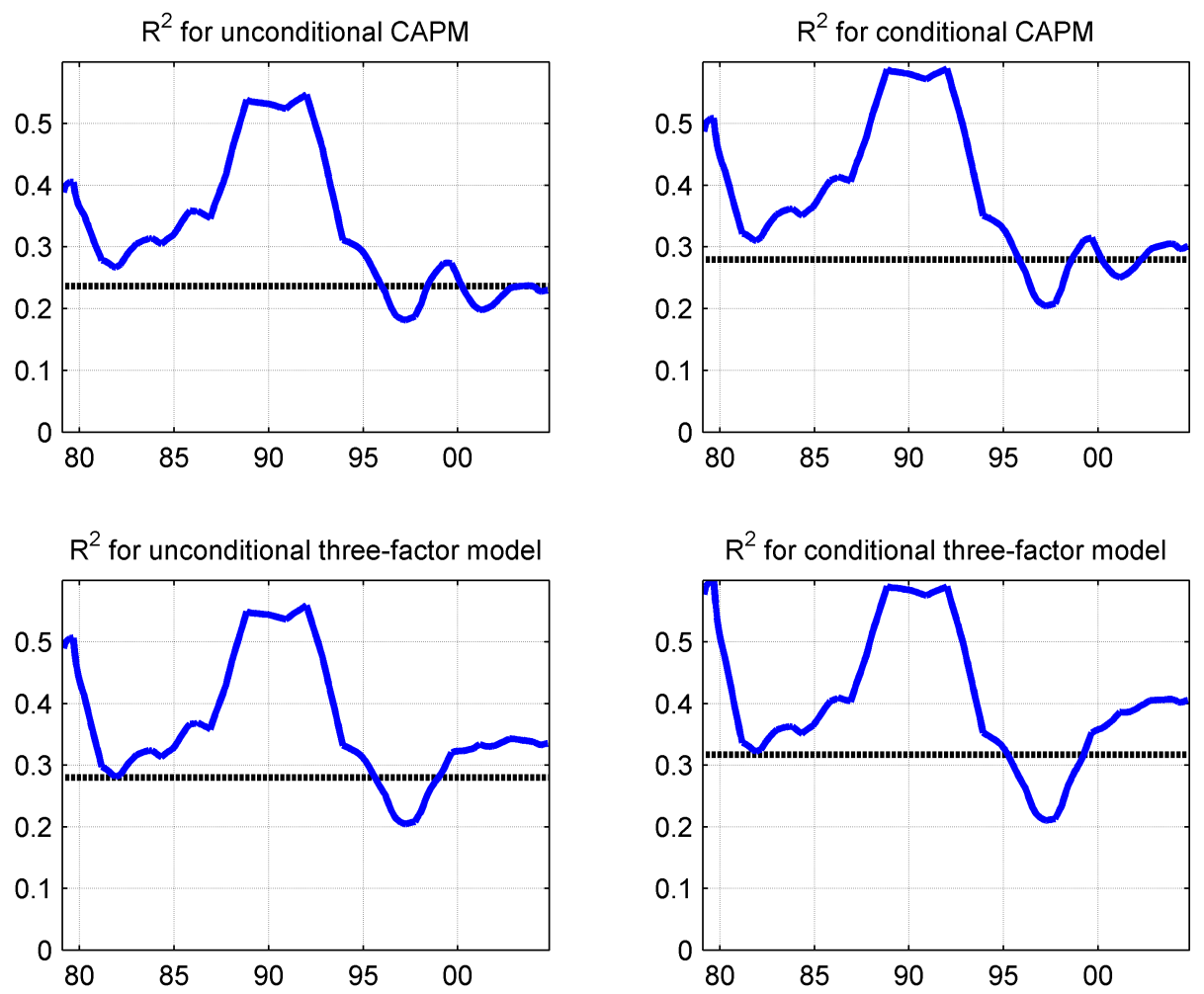

Figure 4: $R^{2}$ for the whole sample period and over a rolling horizon of 60 months for each model

The figure shows the $R^{2}$ for the whole sample period (horizontal line) and over a rolling horizon of 60 months for each model. Over the whole sample period, the $R^{2}$ for the unconditional CAPM is $23.63 \%$, for the conditonal CAPM $27.97 \%$, for the unconditional three-factor model $27.95 \%$, and for the conditional three factor-model $31.69 \%$. 
However, a rolling analysis indicates strong time variation in the explanatory power of asset pricing models. Over a five-year rolling horizon, the explanatory power ranges from approximately $20 \%$ to $60 \%$. All models exhibit similar patterns in their explanatory power across time. In particular, the explanatory power in the time period until 1980 is fairly high (e.g., 40\% for the unconditional CAPM) and drops sharply for the period 19771982 (e.g., $27 \%$ for the unconditional CAPM ). The drop in the empirical performance coincides with the recession in 1980. Afterwards, for all models, the explanatory power increases steadily until 1992 where it reaches a maximum in the observed time period (e.g., $54 \%$ for the unconditional CAPM). After 1992, the empirical performance of all models drops sharply, reaching a temporary low in 1997 (e.g., 18\% for the CAPM) and remains relatively constant until 2004.

The differences in the conditional and unconditional versions of a specific asset pricing model are substantial during economic downturns. During the NBER recessions in the sample period (in 1980, 1990 and 2001), the conditional versions perform better than their unconditional counterparts. In 1980, this difference is about 10\%; in 1990 and 2001 about $5 \%$. This is an indication that factor loadings tend to change during market and economic downturns.

Table 1 shows the root mean squared errors for selected stocks for the unconditional and conditional CAPM and the unconditional and conditional three-factor models. We also report the percentage reduction of the RMSE for the conditional CAPM and the unconditional and the conditional three-factor models.

Overall, at a value of 8.41, the average RMSE for all stocks is highest for the unconditional CAPM. The average RMSE for the conditional CAPM and the unconditional three-factor model is 8.16 and 8.07 , respectively, and therefore slightly lower. This equals a percentage improvement relative to the unconditional CAPM of approximately $3 \%$ and $4 \%$, respectively. The conditional three-factor model reduces the average pricing error by approximately $12 \%$, given a $R M S E$ of 7.38 .

With respect to individual stocks, the data show a large spread between different companies. In particular, the RMSE is high for technology related stocks (such as Cisco, Intel, Oracle, National Semiconductor, Texas Instruments and Time Warner) and for companies facing financial distress such as Aes, EMC, Lucent, Medimumme, and Nextel. 
Table 1: Root mean squared error (RMSE) and relative reduction of RMSE The table shows the RMSE for randomly selected stocks in the cross section for each model and the reduction of the RMSE for the conditional CAPM, the unconditional and conditional three-factor model compared to the unconditional CAPM.

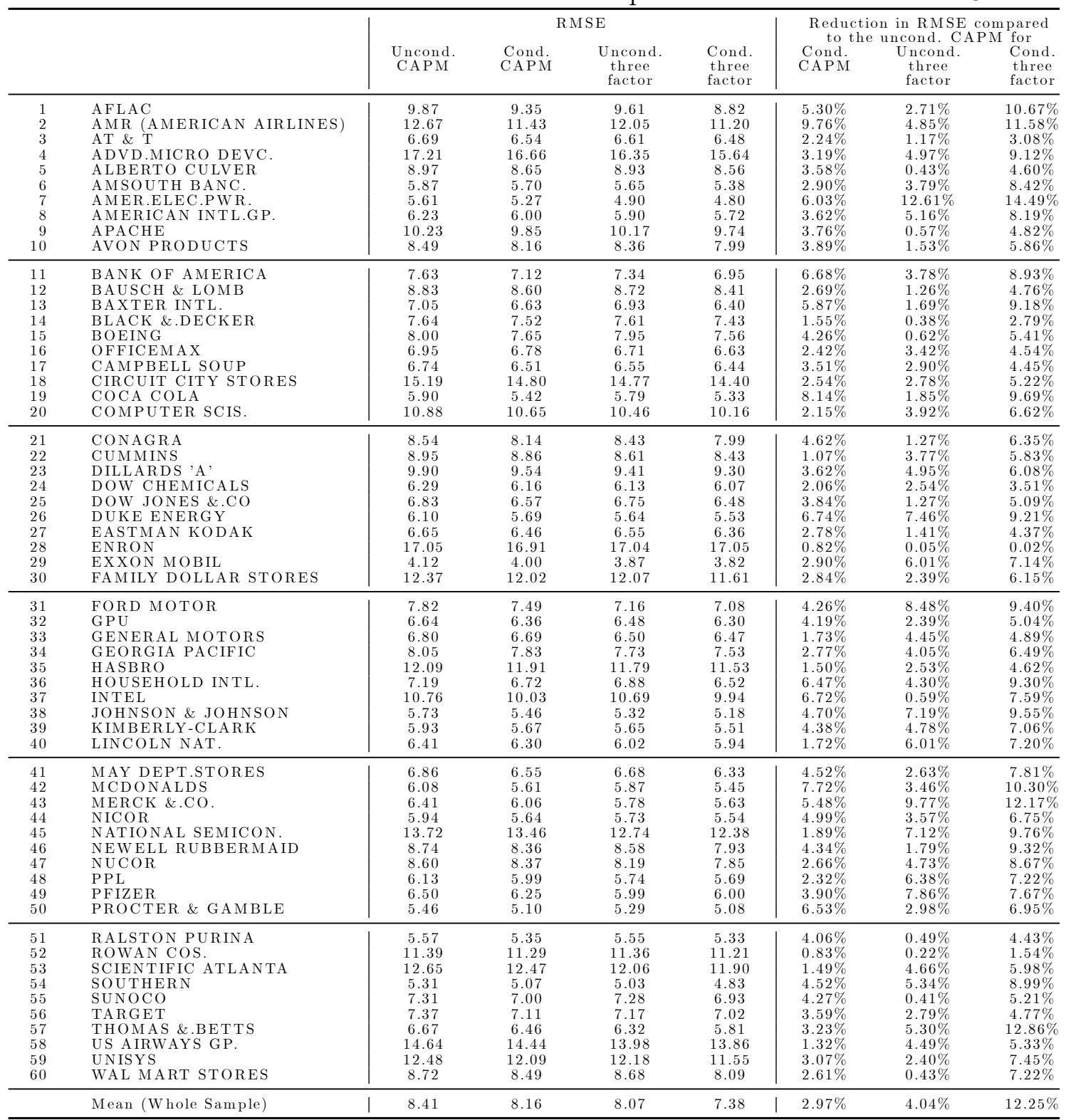




\section{Table 2: Bayes factors}

The table shows the Bayes factors according to the two-step procedure proposed by Charlin and Chib (1995) assuming equal prior probabilities for each model. The number of iterations where the null model was selected, denoted as $p$, is displayed in parenthesis and is used to calculate Bayes factors by $(1-p) / p$. A Bayes factor below 1 supports the null model $M_{0}$ while a Bayes factor larger than 1 supports the alternative model $M_{1}$. In general, a Bayes factor of 1 to 3 is interpreted as weak support for the alternative model and a Bayes factor of 3 to 20 as stronger support for the alternative model (Jeffreys (1961)). Assuming equal prior probabilities for each model, the Bayes factor for model $M_{1}$ compared to $M_{0}$, i.e., $B_{1,0}$, is the reciprocal value of the Bayes factor for model $M_{0}$ compared to $M_{1}$, i.e., $B_{0,1}$. Using the Bayes factors for model selection, the following ranking is obtained: (1) conditional CAPM, (2) conditional three-factor model, (3) unconditional CAPM, (4) unconditional three-factor model.

\begin{tabular}{lc|ccc}
\hline$M_{0}$ & $M_{1}$ & $\begin{array}{c}\text { Conditional } \\
\text { CAPM }\end{array}$ & $\begin{array}{c}\text { Conditional } \\
\text { three-factor }\end{array}$ & $\begin{array}{c}\text { Conditional } \\
\text { three-factor }\end{array}$ \\
\hline Unconditional CAPM & & $4.10(19.6 \%)$ & $0.20(82.81 \%)$ & $1.61(38.4 \%)$ \\
Conditional CAPM & - & $0.56(63.80 \%)$ & $0.44(69.4 \%)$ \\
Unconditional three-factor & & - & - & $2.41(29.3 \%)$ \\
\hline
\end{tabular}

In contrast, the pricing error is low for blue chip companies such as American Express, Du Pont, Exxon, General Electric, and 3M. However, the performance of the models relative to the unconditional CAPM does not show a clear tendency for different groups of firms. In general, the data indicate that asset pricing models work better for mature companies than for companies where firm-individual factors such as growth opportunities and financial distress dominate.

Up to now, the model comparison has focused on traditional measures of fit. The Bayesian framework provides, as shown in Section 2.4, a natural way to compare different models, the so-called second level of inference.

Table 2 shows the estimated Bayes factors for all possible combinations of the four models. The numbers in parentheses denote the percentage $p$ of iterations where the Metropolis-Hastings algorithm selected model $M_{0}$. Assigning equal prior probabilities to each model, i.e., $50 \%$ to $M_{0}$ and $50 \%$ to $M_{1}$, the Bayes factor is given by $(1-p) / p$, as shown by Han \& Carlin (2001).

The Bayes factor for the conditional and unconditional CAPM of 4.10 is evidence in favor of the conditional CAPM and indicates that the conditional CAPM is a better specification than the unconditional CAPM. 
For the unconditional three-factor model the results are somewhat different. The unconditional three-factor model shows, compared to the unconditional CAPM and the conditional CAPM, an inferior performance. The Bayes factors of 0.20 and 0.56 , respectively, indicate that the unconditional three-factor model is misspecified although the RMSE favors the unconditional three-factor model compared to both versions of the CAPM.

The Bayes factors for the conditional three-factor model give an ambiguous result. Compared to the unconditional CAPM and the unconditional three-factor model, the conditional three-factor model shows a superior performance. This is indicated by Bayes factors of 1.61 and 2.41, respectively. However, compared to the conditional CAPM it shows a slightly inferior performance with a Bayes factor of 0.44 .

Overall, the Bayes factors show that the conditional versions of the CAPM and the three-factor model perform better than their unconditional counterparts. The unconditional three-factor model, which does not account for time variation in credit risk and size effects, seems to be misspecified. The conditional three-factor model exhibits a similar performance as the conditional CAPM.

There has been some criticism of the Bayesian paradigm in reaction to Lindley's paradox, such as in Shafer (1982), and Lavine \& Schervish (1999), mainly because flat priors on coefficients are mistakenly thought to be uninformative, but, in fact, they are highly informative. This critisism do not apply in this case because the approach by Carlin \& Chib (1995) is based on a two step procedure, i.e., the posterior estimates for the parameters from the first stage are used to compute Bayes factors in the second stage. The impact of flat priors disappears in this particular setting because the flat priors for the parameters are not used explicitly.

In our analysis, we obtain different rankings depending on the measures used. Using the RMSE, the best-performing model is the conditional three-factor model, followed by the unconditional three-factor model. Conditional CAPM and unconditional CAPM exhibit a slightly inferior performance. The ranking obtained by the $R^{2}$ leaves the first and last place unchanged, but the conditional CAPM is slightly superior to the unconditional three-factor model..Using Bayes factors, however, we obtain the following ranking: (1) conditional CAPM, (2) conditional three-factor model, (3) unconditional CAPM and (4) unconditional three-factor model. 
The fact that traditional measures of fit such as the $R^{2}$ and the $R M S E$ and Bayesian measures of fit such as Bayesian factor deliver contradicting results is not surprising. The main reason is the fundamentally different design of those measures. Bayes factors arise directly from a straightforward application of probability theory, as shown in Section 2.4. Most importantly, whereas $R^{2}$ and $R M S E$ measures always tend to improve with increasing model flexibility, Bayes factors penalize unnecessarily complex models, as shown by Denison et al. (2002) and MacKay (2003). As a consequence, the Bayes factor measure seems a more appropriate measure for model selection than traditional measures.

Our analysis shows that, measured by Bayes factors, both versions of the CAPM are superior to the corresponding versions of the Fama \& French (1993) three-factor model, i.e., the conditional CAPM performs better than the conditional three-factor model and the unconditional CAPM performs better than the unconditional three-factor model. This is evidence that the additional model complexity of the three-factor models is not justified by their empirical performance, although they can explain, in total, a higher proportion of the cross-sectional variation of stock returns. Similar to other authors, such as Ferson \& Harvey (1999), we thus find evidence against the Fama \& French (1993) three-factor model and, in particular, against the unconditional three-factor model.

\section{Conclusion}

We use a new approach for the estimation and the evaluation of unconditional and conditional asset pricing models based on Bayesian inference. Bayesian techniques offer a number of advantages over existing approaches. The Markov Chain Monte Carlo (MCMC) approach accounts for estimation risk, has exact finite-sample properties and does not require the specification of conditioning variables to model time variation in betas. The evaluation approach based on Bayes factors accounts for model uncertainty and the tradeoff between goodness of fit and model complexity.

Using S\&P 500 panel data, we analyze the empirical performance of two popular models, the CAPM and the Fama \& French (1993) three-factor model in their unconditional and conditional versions. Concerning the CAPM, we find that time-variation of betas adds little to the performance if the unconditional CAPM because the $R^{2}$ increases only 
from $23.6 \%$ to $28.0 \%$. Concerning the Fama \& French (1993) three-factor model, we find that time-variation of the factor loadings on the small-minus-big (SMB) factor and the high-minus-low (HML) factor increases the explained variance by a similar magnitude; the $R^{2}$ improves from $28.0 \%$ to $31.7 \%$. Our analysis indicates that firm-specific exposure to systematic market risk, credit risk, measured by the HML factor, and to systematic size effects, measured by the SMB factor, shows a significant degree of variability. Using traditional measures of fit, our findings are comparable to Ferson \& Harvey (1999) and Jagannathan \& Wang (1996).

A Bayesian model comparison, however, delivers a more differentiated result. The Bayes factors show that the conditional asset pricing models exhibit better performance than their unconditional counterparts. The performance of the conditional CAPM is slightly superior to the performance of the conditional Fama \& French (1993) three-factor model. Our results show that, as in Jagannathan \& Wang (1996), the conditional CAPM can explain the cross section of stock returns much better than a static CAPM. However, the use of more advanced model selection criteria such as the Bayes factors indicate that the better explanatory power gained from more flexible conditional models, if measured by $R^{2}$, is not very robust. 


\section{Notes}

\footnotetext{
${ }^{1}$ The use of the realized risk premium is a standard assumption in testing asset pricing model (see e.g. Fama \& MacBeth (1973), Gibbons et al. (1989), Campbell, Lo \& MacKinlay (1997)). However, some authors have criticized this approach ("unobservability of the market portfolio", see e.g. Roll (1977)), but the use of the CRSP market portfolio as a proxy for the market return is a standard approach used also in Fama \& French (1996), Ferson \& Harvey (1999), and Wang (2003).

${ }^{2}$ However, it is straightforward to incorporate alternative specifications for the beta process in our approach. In particular, we tested for alternative specifications of the $\beta$ process, e.g., by using a $A R(1)$ term and a mean reverting term. The results are very robust with respect to these two alternative specifications. In particular, the $A R(1)$ process showed that the behaviour of betas is extremely close to a unit root.

${ }^{3}$ To ensure robustness, a number of alternative specifications of prior distributions have been tested. For example, we used for the variance a standard inverse gamma distribution. The results did not differ.

${ }^{4}$ Therefore, a survival bias cannot be excluded.

${ }^{5}$ According to NBER, the recession dates in the sample period are (1) November 1973 to March 1975, (2) January 1980 to July 1980, (3) July 1981 to November 1982, (4) July 1990 to March 1991, and (5) March 2001 to November 2001.
}

\section{References}

Akaike, H. (1980), Likelihood and the Bayes procedure, in J. Bernardo, M. DeGroot, D. Lindley \& A. Smith, eds, 'Bayesian Statistics', University Press, Valencia, Spain, pp. $143-166$.

Avramov, D. \& Chao, J. C. (2006), 'An exact Bayes test of asset pricing models with application to international markets', Journal of Business 79, 293-323.

Banz, R. (1981), 'The relationship between return and market value of common stocks', Journal of Financial Economics 9, 3-18.

Basu, S. (1977), 'The investment performance of common stocks in relation to their priceearnings ratios: A test of the efficient market hypothesis', Journal of Finance 32, 663682.

Bauwens, L., Lubrano, M. \& Richard, J.-F. (1999), Bayesian Inference in Dynamic Econometric Models, Oxford University Press, Oxford. 
Berglund, T. \& Knif, J. (1999), 'Accounting for the accuracy of beta estimates in CAPM test on assets with time-varying risks', European Financial Management 5, 29-42.

Braun, P., Nelson, D. \& Sunier, A. (1995), 'Good news, bad news, volatility and betas', Journal of Finance 50, 1575-1604.

Campbell, J., Lo, A. \& MacKinlay, A. (1997), The Econometrics of Financial Markets, Princeton University Press, Princeton.

Carlin, B. P. \& Chib, S. (1995), 'Bayesian model choice via Markov Chain Monte Carlo methods', Journal of the Royal Statistical Society. Series B. 57, 473-484.

Carlin, B. P. \& Louis, T. A. (2000), Bayes and Empirical Bayes Methods for Data Analysis, Chapman \& Hall / CRC, New York.

Carlin, B. P., Polson, N. G. \& Stoffer, D. S. (1992), 'A Monte Carlo approach to nonnormal and nonlinear state-space modeling', Journal of the American Statistical Association 87, 493-500.

Chan, L. K., Hamao, Y. \& Lakonishok, J. (1991), 'Fundamentals and stock returns in Japan', Journal of Finance 46, 1739-1765.

Congdon, P. (2001), Bayesian Statistical Modelling, John Wiley \& Sons, New York.

Congdon, P. (2003), Applied Bayesian Modelling, John Wiley \& Sons, New York.

Cowles, M. K. (2004), 'Review of WinBUGS 1.4', American Statistician 58, 330-336.

Denison, D. G., Holmes, C. C., Mallick, B. K. \& Smith, A. F. (2002), Bayesian Methods for Nonlinear Classification and Regression, John Wiley \& Sons, New York.

Fama, E. F. \& French, K. R. (1996), 'Multifactor explanations of asset pricing anomalies', Journal of Finance 51, 55-84.

Fama, E. F. \& MacBeth, J. D. (1973), 'Risk, return, and equilibrium: Empirical tests', Journal of Political Economy 81, 607-636.

Fama, E. \& French, K. (1992), 'The cross-section of expected stock returns', Journal of Finance 47, 427-465. 
Fama, E. \& French, K. (1993), 'Common risk factors in the returns on stocks and bonds', Journal of Financial Economics 33, 3-57.

Ferson, E. \& Koraczyk, R. (1995), 'Do arbitrage pricing models explain the predictability of stock returns', Journal of Business 68, 309-349.

Ferson, W. \& Foerster, S. (1994), 'Finite sample properties of the generalized methods of moments in tests of conditional asset pricing models', Journal of Financial Economics 36, 29-35.

Ferson, W. \& Harvey, C. (1991), 'The variation of economic risk premiums', Journal of Political Economy 99, 385-415.

Ferson, W. \& Harvey, C. (1999), 'Conditioning variables and the cross section of stock returns', Journal of Finance 54, 1325-1360.

Geman, S. \& Geman, D. (1984), 'Stochastic relaxation, Gibbs distributions and the Bayesian restoration of images', IEEE Transactions on Pattern Analysis and Machine Intelligence 6, 721-741.

Geweke, J. \& Zhou, G. (1996), 'Measuring the pricing error in the arbitrage pricing theory', Review of Financial Studies 9, 557-587.

Ghysels, E. (1998), 'On stable factor structures in the pricing of risk: Do time-varying betas help or hurt?', Journal of Finance 53, 549-574.

Gibbons, M. R., Ross, S. A. \& Shanken, J. (1989), 'A test of the efficiency of a given portfolio', Econometrica 57, 1121-1152.

Han, C. \& Carlin, B. P. (2001), 'Markov Chain Monte Carlo methods for computing Bayes factors: A comparative review', Journal of the American Statistical Association 96, $1122-1132$.

Hansen, L. \& Richard, S. (1987), 'The role of conditioning information in deducing testable restrictioncs implied in dynamic asset pricing models', Econometrica 55, 587-613.

Harvey, C. \& Zhou, G. (1990), 'Bayesian inference in asset pricing tests', Journal of Financial Economics 26, 221-254. 
Hastings, W. (1970), 'Monte Carlo sampling methods using Markov Chains and their application', Biometrica 57, 97-109.

Heston, S. L., Rouwenhorst, K. G. \& Wessels, R. E. (1999), 'The role of beta and size in the cross-section of European stock returns', European Financial Management $5,9-27$.

Jagannathan, R. \& Wang, Z. (1996), 'The conditional CAPM and the cross section of expected returns', Journal of Finance 51, 3-52.

Jeffreys, H. (1961), Theory of Probability, Oxford University Press, Oxford.

Kass, R. E. \& Raftery, A. E. (1995), 'Bayes factors', Journal of the American Statistical Association 90, 773-795.

Kim, C.-J. \& Nelson, C. R. (1999), State-Space Models with Regime Switching, MIT Press, Cambridge, Massachusetts.

Kitagawa, G. \& Gersch, W. (1996), Smoothness Priors Analysis of Time Series, Springer, New York.

Koutmos, G. \& Knif, J. (2002), 'Estimating systematic risk using time varying distributions', European Financial Management 8, 59-73.

Lavine, M. \& Schervish, M. J. (1999), 'Bayes factors: What they are and what they are not', American Statistician 53, 119-122.

MacKay, D. J. C. (2003), Information Theory, Inference $\mathcal{G}$ Learning Algorithms, Cambridge University Press, Cambridge.

McCulloch, R. \& Rossi, P. (1991), 'A Bayesian approach to testing the arbitrage pricing theory', Journal of Econometrics 49, 141-168.

Metropolis, N. \& Ulam, S. (1949), 'The Monte Carlo method', Journal of the American Statistical Association 19, 425-442.

Neal, R. (2003), 'Slice sampling', Annals of Statistics 31, 705-741. 
Petrella, G. (2005), 'Are Euro area small cap stocks an asset class? evidence from meanvariance spanning tests', European Financial Management 11, 229-253.

Robert, C. R. \& Casella, G. (1999), Monte Carlo Statistical Methods, Springer, New York.

Robert, C. R. \& Casella, G. (2005), Monte Carlo Statistical Methods, 2nd edn, Springer, New York.

Roll, R. (1977), 'A critique of the asset pricing theory's tests', Journal of Financial Economics 4, 129-176.

Shafer, G. (1982), 'Lindley's paradox', Journal of the American Statistical Society 77, 325351.

Shanken, J. (1987), 'A Bayesian approach to testing portfolio efficiency', Journal of Financial Economics 19, 195-215.

Wang, K. (2003), 'Asset pricing with conditioning information: A new test', Journal of Finance 58, 161-196.

Zellner, A. (1971), An Introduction to Bayesian Inference in Econometrics, John Wiley \& Sons, New York. 
5 Appendix 


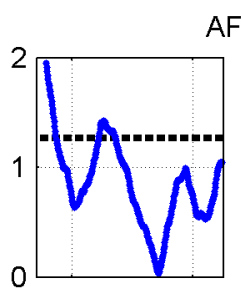

AFLAC (MRP, HML, SMB)
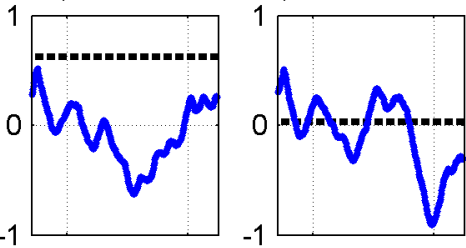

AT \& $T(M R P, H M L, S M B)$
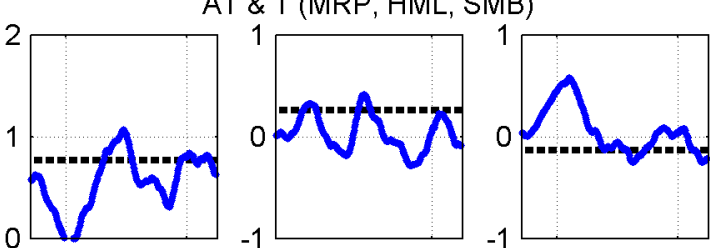

ALBERTO CULVER (MRP, HML, SMB)
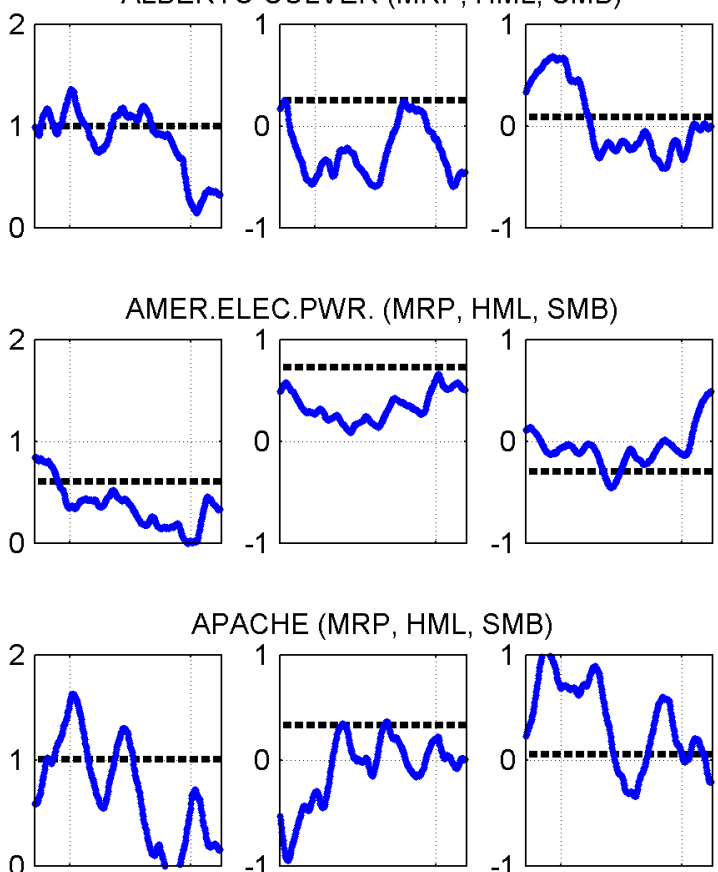

APACHE (MRP, HML, SMB)
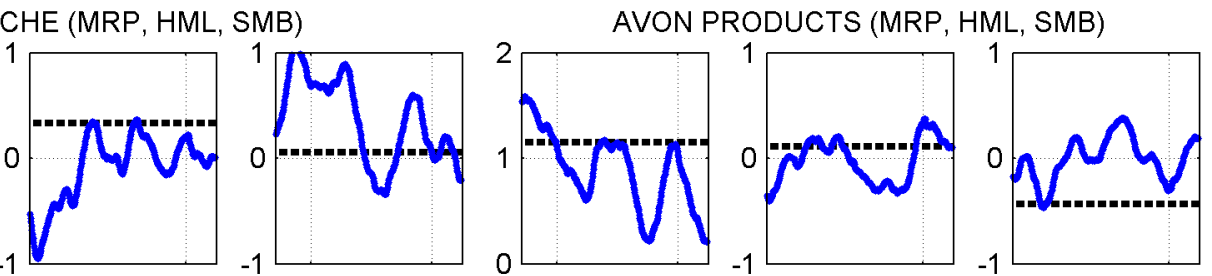

Figure A1: Estimated factor loadings for selected stocks for the Fama and French three-factor model

The figure shows estimated factor loadings for selected stocks for the Fama and French three-factor model, i.e., the factor loadings on the market risk premium (MRP), the value premium (HML), and the size premium (SMB). Sample period: January 1974 to August 2004. 

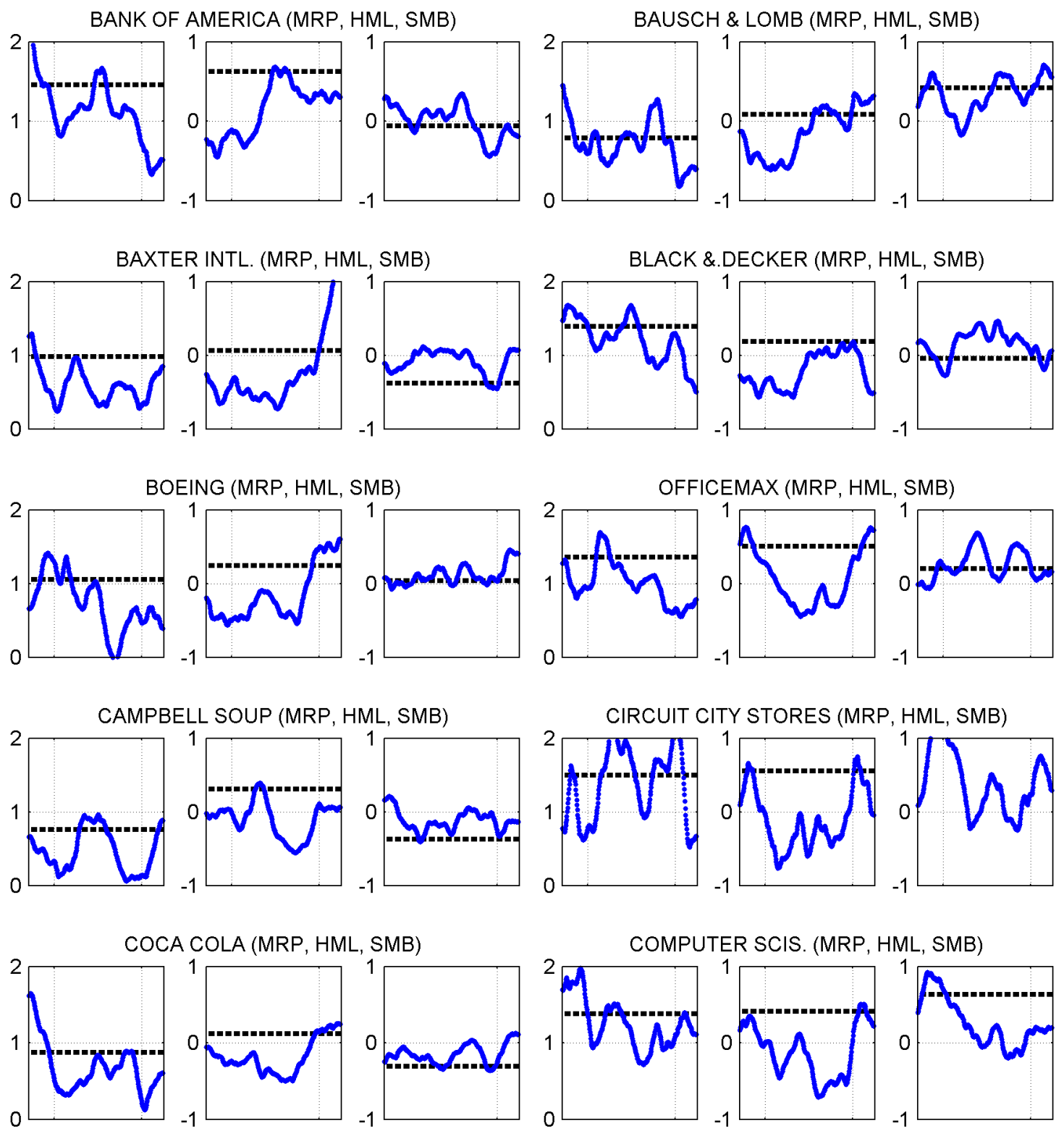

Figure A2: Estimated factor loadings for selected stocks for the Fama and French three-factor model

The figure shows estimated factor loadings for selected stocks for the Fama and French three-factor model, i.e., the factor loadings on the market risk premium (MRP), the value premium (HML), and the size premium (SMB). Sample period: January 1974 to August 2004. 

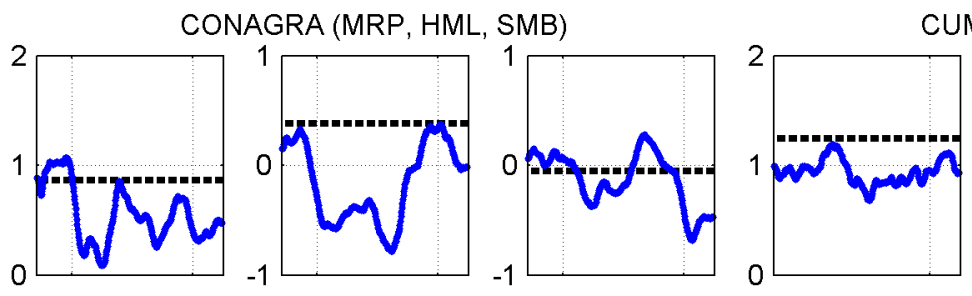

CUMMINS (MRP, HML, SMB)
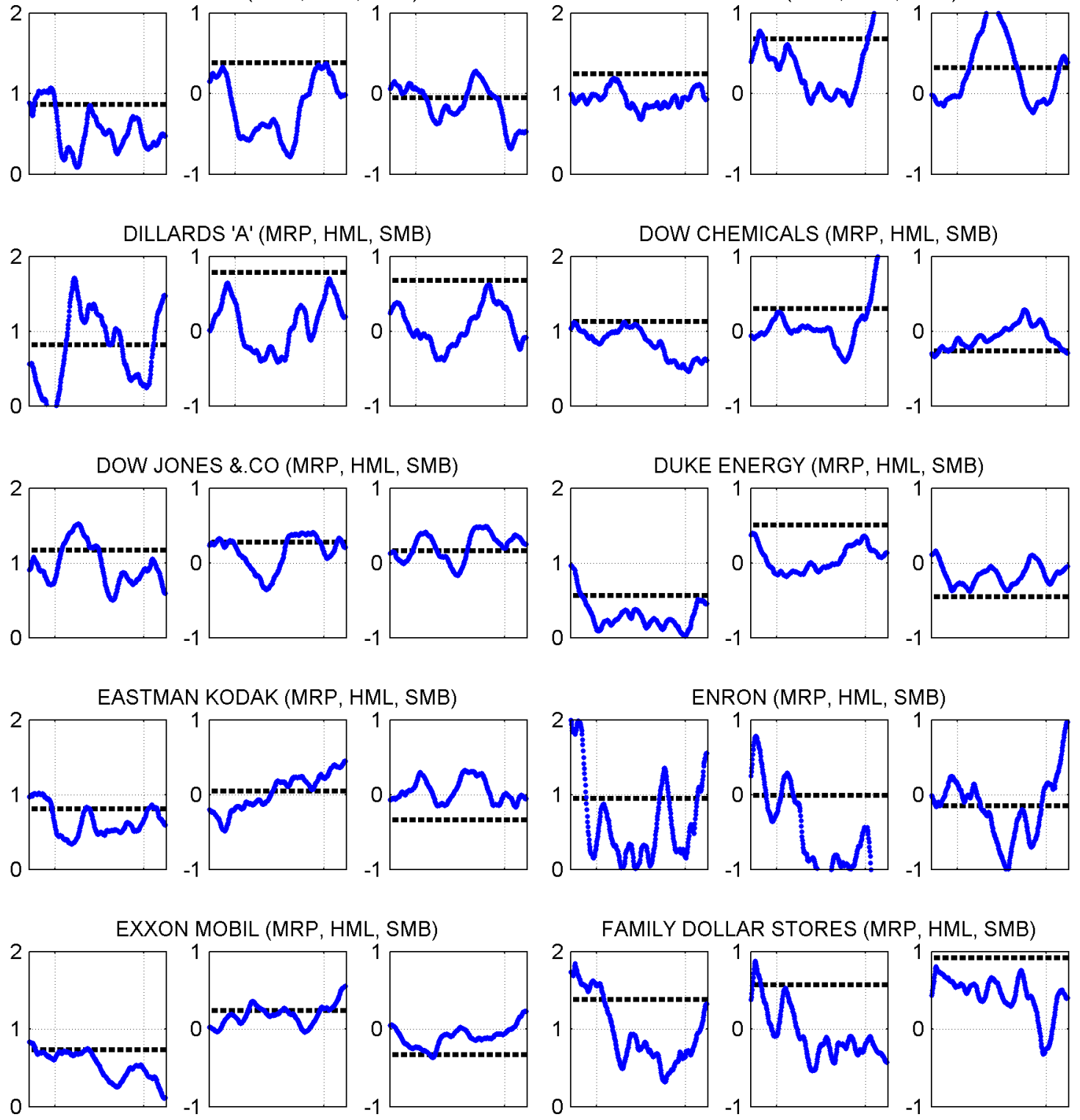

Figure A3: Estimated factor loadings for selected stocks for the Fama and French three-factor model

The figure shows estimated factor loadings for selected stocks for the Fama and French three-factor model, i.e., the factor loadings on the market risk premium (MRP), the value premium (HML), and the size premium (SMB). Sample period: January 1974 to August 2004 . 

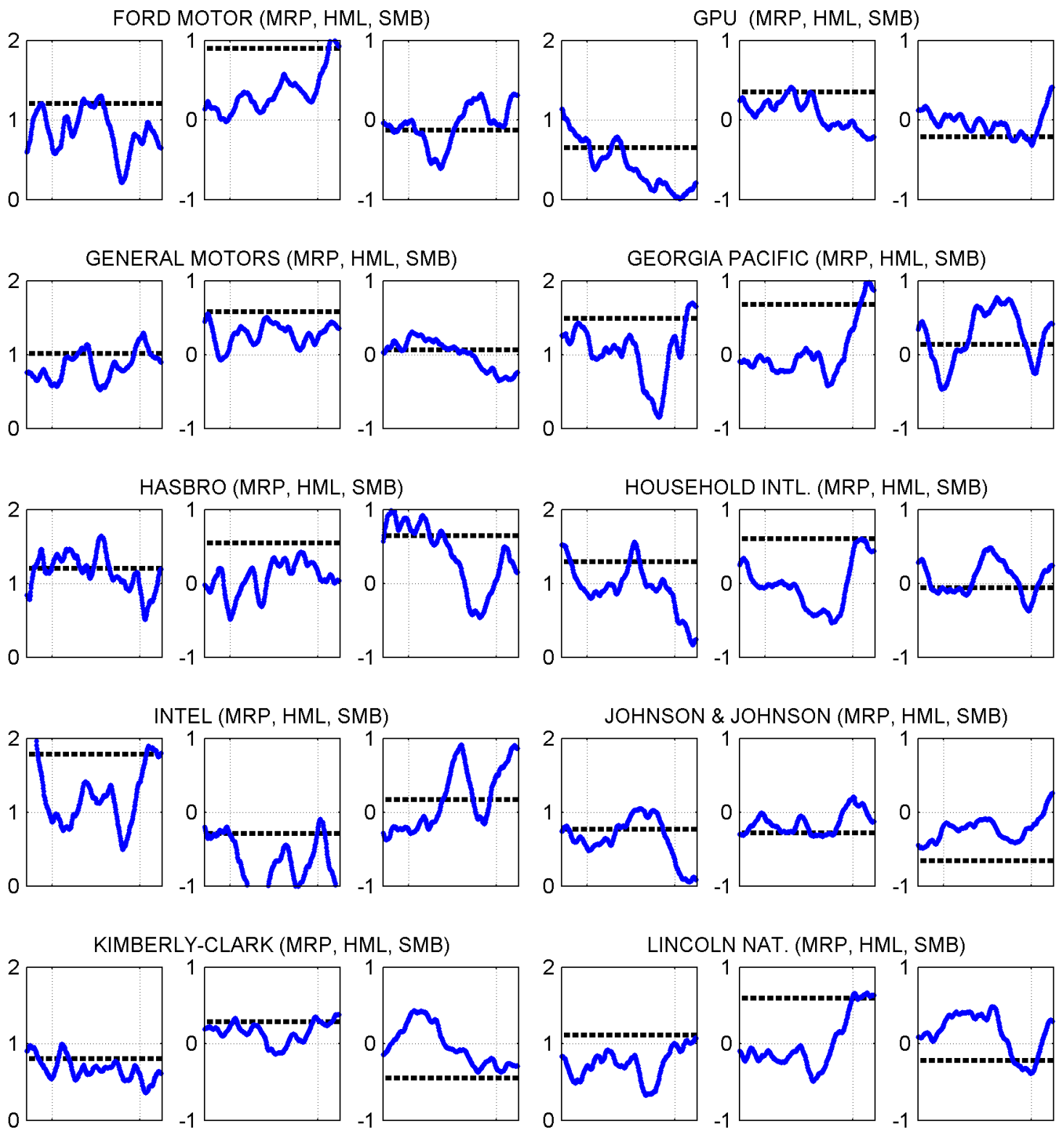

Figure A4: Estimated factor loadings for selected stocks for the Fama and French three-factor model

The figure shows estimated factor loadings for selected stocks for the Fama and French three-factor model, i.e., the factor loadings on the market risk premium (MRP), the value premium (HML), and the size premium (SMB). Sample period: January 1974 to August 2004. 

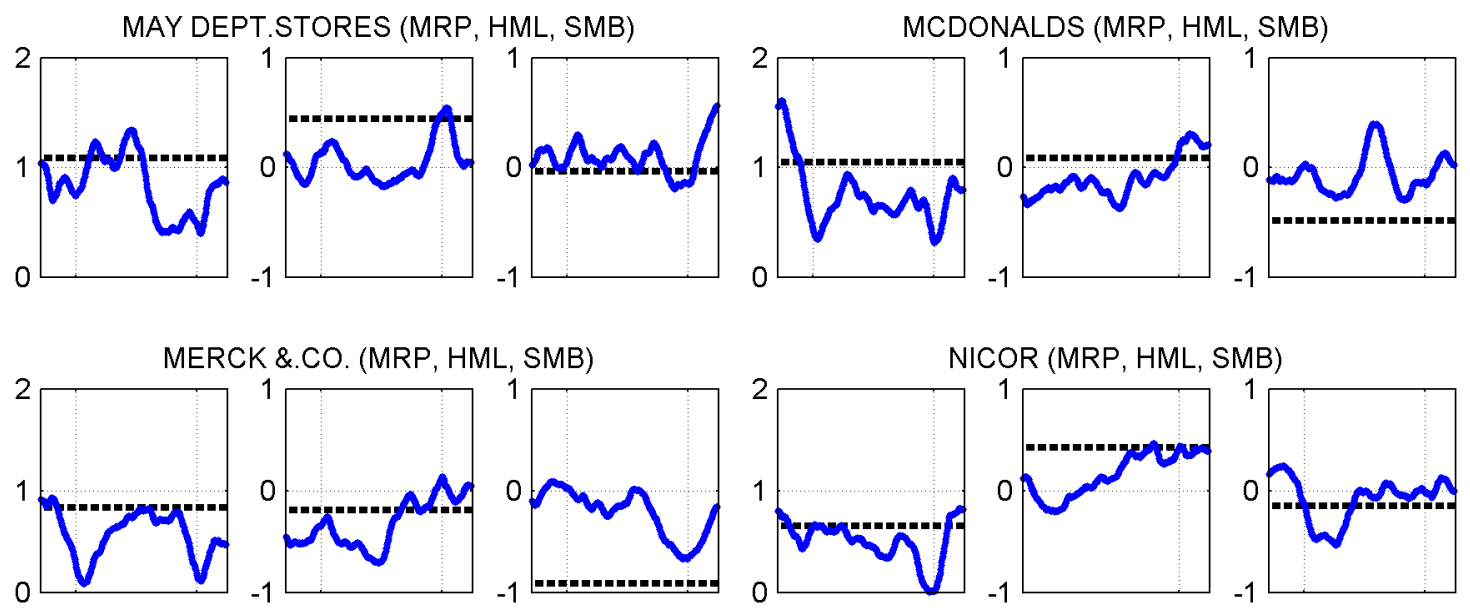

NICOR (MRP, HML, SMB)
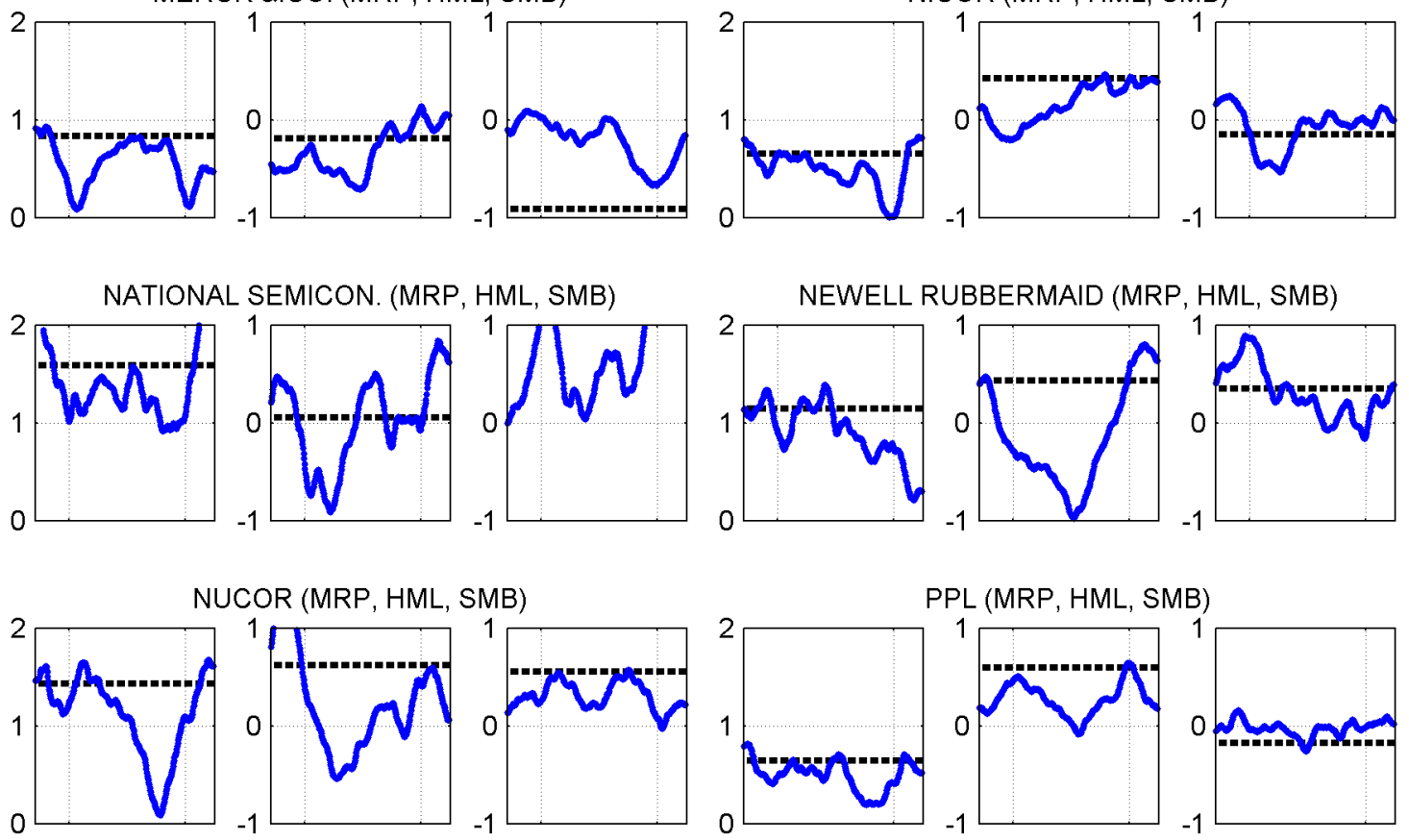

PPL (MRP, HML, SMB)
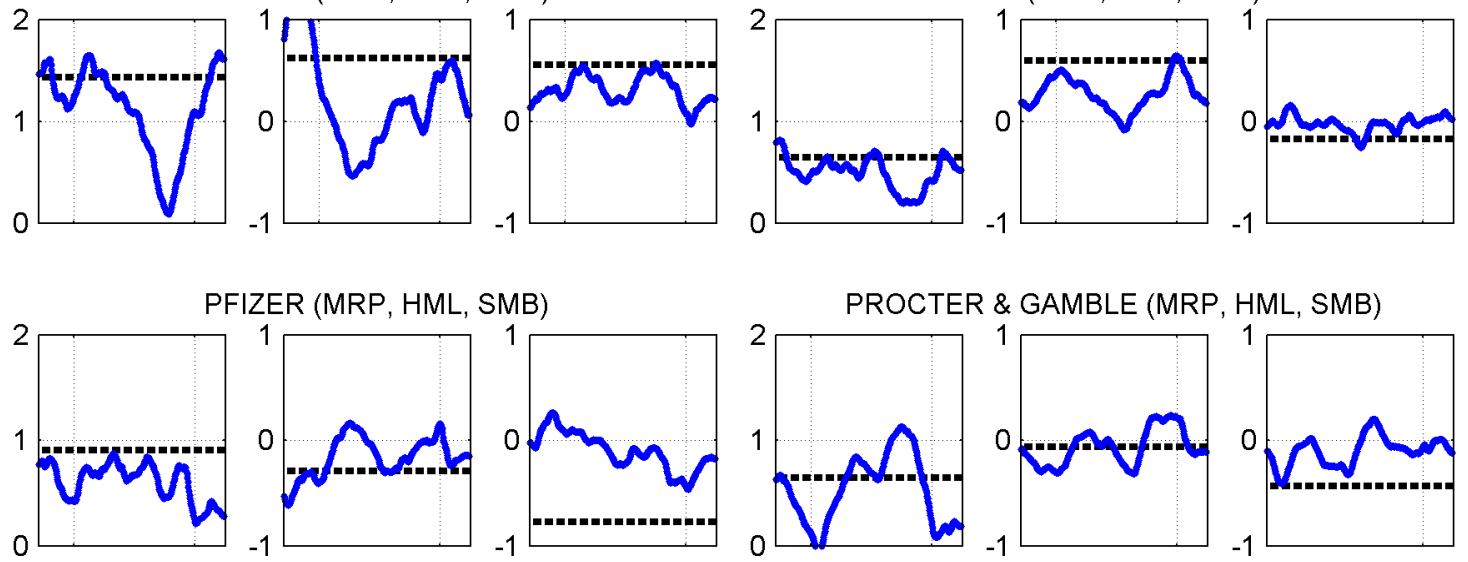

Figure A5: Estimated factor loadings for selected stocks for the Fama and French three-factor model

The figure shows estimated factor loadings for selected stocks for the Fama and French three-factor model, i.e., the factor loadings on the market risk premium (MRP), the value premium (HML), and the size premium (SMB). Sample period: January 1974 to August 2004. 

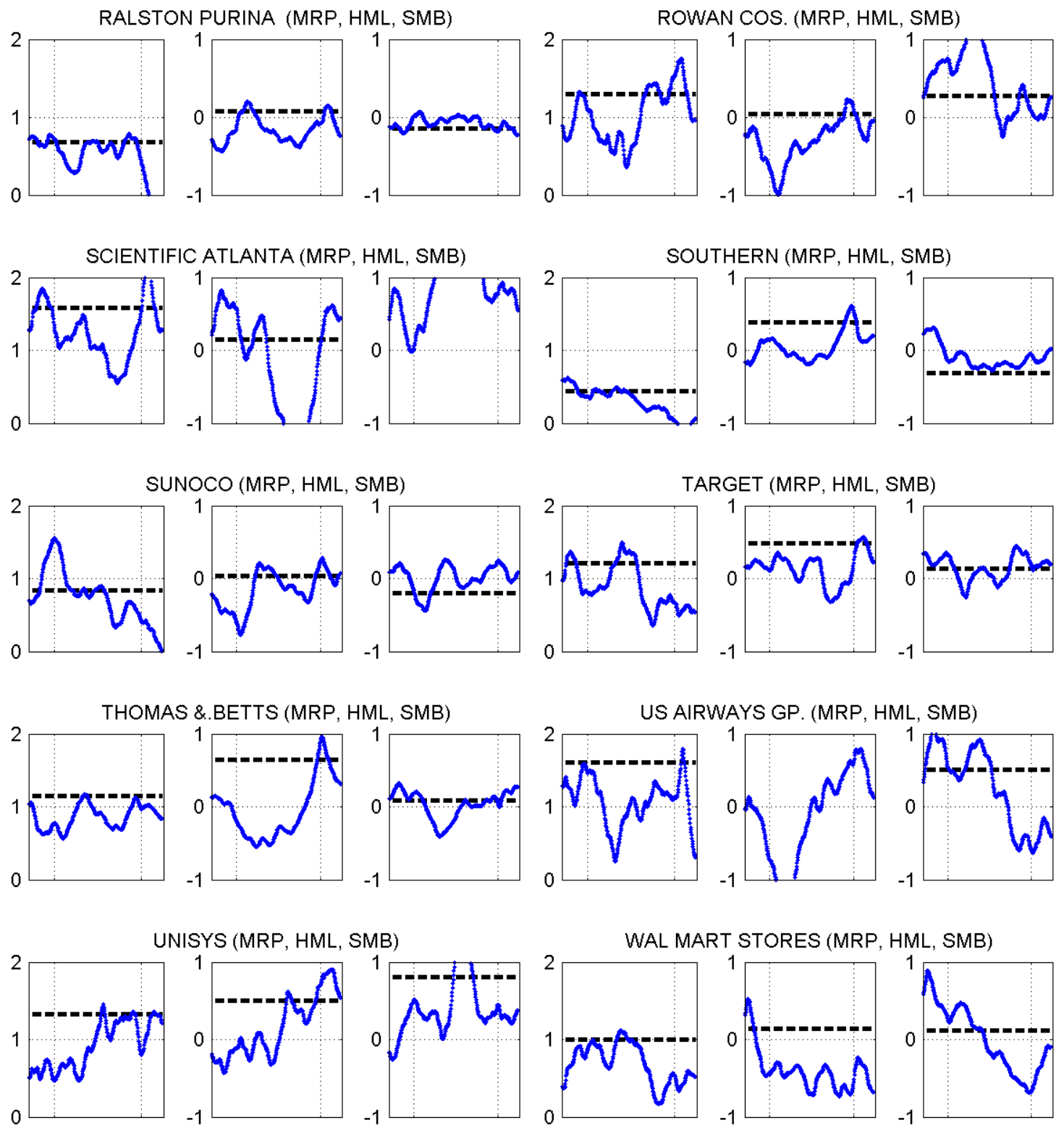

Figure A6: Estimated factor loadings for selected stocks for the Fama and French three-factor model

The figure shows estimated factor loadings for selected stocks for the Fama and French three-factor model, i.e., the factor loadings on the market risk premium (MRP), the value premium (HML), and the size premium (SMB). Sample period: January 1974 to August 2004 . 